ARTICLE

Received 20 Feb 2014 | Accepted 4 Apr 2014 | Published 13 May $2014 \quad$ DOl: 10.1038/ncomms4812

\title{
Chd5 orchestrates chromatin remodelling during sperm development
}

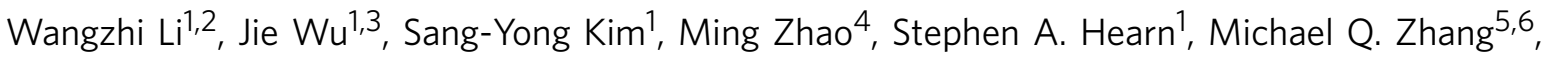
Marvin L. Meistrich ${ }^{4} \&$ Alea A. Mills ${ }^{1}$

One of the most remarkable chromatin remodelling processes occurs during spermiogenesis, the post-meiotic phase of sperm development during which histones are replaced with sperm-specific protamines to repackage the genome into the highly compact chromatin structure of mature sperm. Here we identify Chromodomain helicase DNA binding protein 5 (Chd5) as a master regulator of the histone-to-protamine chromatin remodelling process. Chd5 deficiency leads to defective sperm chromatin compaction and male infertility in mice, mirroring the observation of low CHD5 expression in testes of infertile men. Chd5 orchestrates a cascade of molecular events required for histone removal and replacement, including histone $4(\mathrm{H} 4)$ hyperacetylation, histone variant expression, nucleosome eviction and DNA damage repair. Chd5 deficiency also perturbs expression of transition proteins (Tnp1/Tnp2) and protamines (Prm1/2). These findings define Chd5 as a multi-faceted mediator of histoneto-protamine replacement and depict the cascade of molecular events underlying this process of extensive chromatin remodelling.

\footnotetext{
${ }^{1}$ Cold Spring Harbor Laboratory, Cold Spring Harbor, New York 11724, USA. ${ }^{2}$ Molecular and Cellular Biology Program, Stony Brook University, Stony Brook, New York 11794, USA. ${ }^{3}$ Department of Applied Mathematics and Statistics, Stony Brook University, Stony Brook, New York 11794, USA. ${ }^{4}$ Department of Experimental Radiation Oncology, MD Anderson Cancer Center, Houston, Texas 77030, USA. ${ }^{5}$ Department of Molecular and Cell Biology, Center for Systems Biology, The University of Texas at Dallas, Richardson, Texas 75080, USA. ${ }^{6}$ MOE Key Laboratory of Bioinformatics and Bioinformatics Division, Center for Synthetic and System Biology, TNLIST/Department of Automation, Tsinghua University, Beijing 100084, China. Correspondence and requests for materials should be addressed to A.A.M. (email: mills@cshl.edu).
} 
S permatogenesis is an intricate biological process that transforms diploid spermatogonial stem cells into haploid spermatozoa in seminiferous tubules of testis. It consists of three major phases: mitosis, meiosis and spermiogenesis ${ }^{1}$. Spermatogonial stem cells first multiply by repeated rounds of mitosis and differentiate into primary spermatocytes, which subsequently undergo meiosis and become haploid round spermatids. Round spermatids then mature into highly specialized spermatozoa through spermiogenesis, the final phase of spermatogenesis ${ }^{1}$. During spermiogenesis, round spermatids undergo a number of characteristic changes including elongation and condensation of the nucleus, formation of the acrosome and flagellum, and removal of cytoplasm ${ }^{2}$. In mouse, spermatogenesis is subdivided into twelve stages (stages I-XII), whereas spermiogenesis is further divided into 16 steps (steps 1-16) mainly defined by changes in acrosome structure and nuclear morphology of the maturing spermatids ${ }^{1,3-5}$

Extensive chromatin remodelling occurs during spermiogenesis, which results in the majority of nucleosomal histones being replaced by sperm-specific basic proteins, initially transition proteins and ultimately protamines ${ }^{6}$. Protamines are distinct from histones, packaging the sperm genome into a distinct toroid chromatin structure 7 . This dramatic histone-to-protamine remodelling repackages the sperm genome into a chromatin structure that is sixfold or more compact than that of somatic cells, and is essential for normal sperm development ${ }^{6,8}$. Given its extensive degree of chromatin remodelling, spermiogenesis offers a unique process to study mechanisms of chromatin remodelling. However, this process is currently understudied and still poorly understood, mainly due to the complexity of the process itself and lack of in vitro experimental systems for studying it. In particular, chromatin remodellers are believed to be essential for facilitating the extensive degree of chromatin remodelling during spermiogenesis, but their roles in this process are not well elucidated. In this study, we discovered that Chromodomain helicase DNA-binding protein 5 (Chd5) plays an orchestrating role in the histone-to-protamine remodelling process during spermiogenesis. Chd5 is a member of the CHD family of chromatin remodellers, which we identified as a dosage-sensitive tumour suppressor ${ }^{9}$. While recent studies reveal that Chd5 binds unmodified histone $3(\mathrm{H} 3)$ via its dual plant homeodomains ${ }^{10,11}$ and that this interaction is essential for tumour suppression ${ }^{10}$, the ability of Chd5 to mediate chromatin dynamics in the context of normal cells is not well understood. We find that Chd5 is highly expressed during spermiogenesis and plays essential roles during sperm development. Inactivation of Chd5 in mice leads to sperm chromatin compaction defects and male infertility. We reveal that Chd5 both mediates a cascade of molecular events for histone removal and modulates the homeostasis of transition proteins and protamines, identifying Chd5 as a master regulator of the histone-to-protamine chromatin remodelling process during spermiogenesis.

\section{Results}

Chd5 is expressed in spermatids during spermiogenesis. Using immunofluorescence analyses with a previously validated antibody specific for Chd5 (refs 10,12) we found that Chd5 is expressed in mouse testes specifically during spermiogenesis (Fig. 1, Supplementary Fig. 1). Chd5 was first detectable after meiosis, when it was expressed within nuclei of step 4 spermatids (Fig. 1). At this phase, Chd5 was weakly expressed throughout the nucleus but was highly expressed in an intense focal spot near the chromocentre, a cluster of centromeres and pericentromeric heterochromatin ${ }^{13}$. Chd5 expression peaked at steps $7-8$, when it was expressed robustly throughout the nucleus and was enriched in the chromocentre, where Chd5 colocalized with the heterochromatin mark $\mathrm{H} 3 \mathrm{~K} 9 \mathrm{me} 3$, and was expressed in a pattern similar to that of the repressive histone mark H3K27me3 (Fig. 1b, Supplementary Fig. 2). Chd5 expression decreased after step 9, when it remained enriched in heterochromatin, and was not detectable after step 10 (Fig. 1). The Chd5-intense focal spot juxtaposed to the edge of the chromocentre was present in round spermatids from steps $4-8$, and was positioned at the junction between the chromocentre and the post-meiotic sex chromosome, both of which are DAPI-intense sub-nuclear structures within spermatids (Fig. 1, Supplementary Fig. 3a) ${ }^{14}$. In contrast to the chromocentre, the Chd5-intense focal spot was DAPI-weak and was negative for $\mathrm{H} 3 \mathrm{~K} 9 \mathrm{me} 3$ (Fig. 1b, Supplementary Fig. 3a), suggesting that it marks transcriptionally active chromatin. We speculated that the Chd5-intense foci may be within nucleoli. Coimmunostaining of Chd5 with the nucleolar marker fibrillarin showed that the Chd5-intense spot was near the nucleolus in many spermatid nuclei, but was clearly separated in others (Supplementary Fig. 3b), raising the possibility that Chd5 transiently associates with the nucleolus. These findings indicate that Chd5 is expressed specifically in nuclei of round and early elongating spermatids during spermiogenesis where it is primarily enriched in heterochromatic regions, and that Chd5 is also expressed in an intense focal spot in a non-heterochromatic region juxtaposed to the chromocentre.

Chd5 deficiency impairs sperm development and fertility. The dynamics of Chd5 expression during spermiogenesis indicated that it might play a functional role in chromatin remodelling during spermatid maturation. To explore this possibility, we generated Chd5-deficient mice carrying the Chd5 $5^{\text {Aam1 }}$ null allele (Supplementary Fig. 4). Western blot analyses using a validated antibody ${ }^{10}$ recognizing a part of Chd5 protein not disrupted by gene targeting demonstrated that the Chd5 protein was not detected in testes of Chd5 $5^{\text {Aaml }}$ homozygotes (Fig. 2a). Chd5 $5^{\text {Aam } 1-1-}$ mice were viable and grossly normal. Mating tests revealed that whereas Chd $5^{\text {Aam } 1-}{ }^{-}$female mice and Chd5 $5^{\text {Aam } 1+/-}$ mice of both genders were fertile, Chd5 $5^{\text {Aam } 1-/-}$ males were either sub-fertile or sterile (Supplementary Table 1). Chd $5^{\text {Aam } 1-1-}$ mice had significantly lower sperm counts, and

Figure 1 | Chd5 is expressed in step 4-10 spermatids and is enriched in heterochromatin during spermiogenesis. Roman numerals indicate the spermatogenic stages of the tubules in wild-type testes sections. (a) Blue, DAPI; green, Chd5; RS, round spermatid; ES, elongating spermatid; ECS, elongating and condensing spermatid; CS, condensed spermatid; $\mathrm{P}$, pachytene spermatocyte; Mi, meiotic division. Arrow heads mark the chromocentre. Scale bar, $10 \mu \mathrm{m}$. (b) Chd5 is enriched in DAPI-intense heterochromatic regions, and colocalizes with heterochromatin marker H3K9me3. Top panel, step 7-8 round spermatids; bottom panel, step 9-10 elongating spermatids. Arrow heads mark the chromocentre. Scale bar, $5 \mu \mathrm{m}$. (c) Schematic of Chd5 expression during spermatogenesis. Spermatogenesis is divided into twelve stages (stage I-XII) in mouse and each stage has a distinct cellular composition. Spermiogenesis, the maturation process of haploid spermatids, is divided into 16 steps (steps 1-16). Green marks Chd5 protein expression. Chd5 is specifically expressed in spermatids from steps 4-10, with peak expression in step 7-8 round spermatids, where Chd5 is enriched in the heterochromatic chromocentre. A focus of intense Chd5 protein expression is located adjacent to the junction of the chromocentre and the post-meiotic sex chromosomes in steps 4-8 spermatids. Spermatogonia (A, In, B); spermatocyte (PI, preleptotene; L, leptotene; Z, zygotene; P, pachytene; D, diakinesis; $\mathrm{Mi}$, meiotic division); $\mathrm{Ag}$, acrosomic granule; Ac, acrosomic cap. The diagram is drawn based on the illustration of Hess et al. ${ }^{71}$ 
a

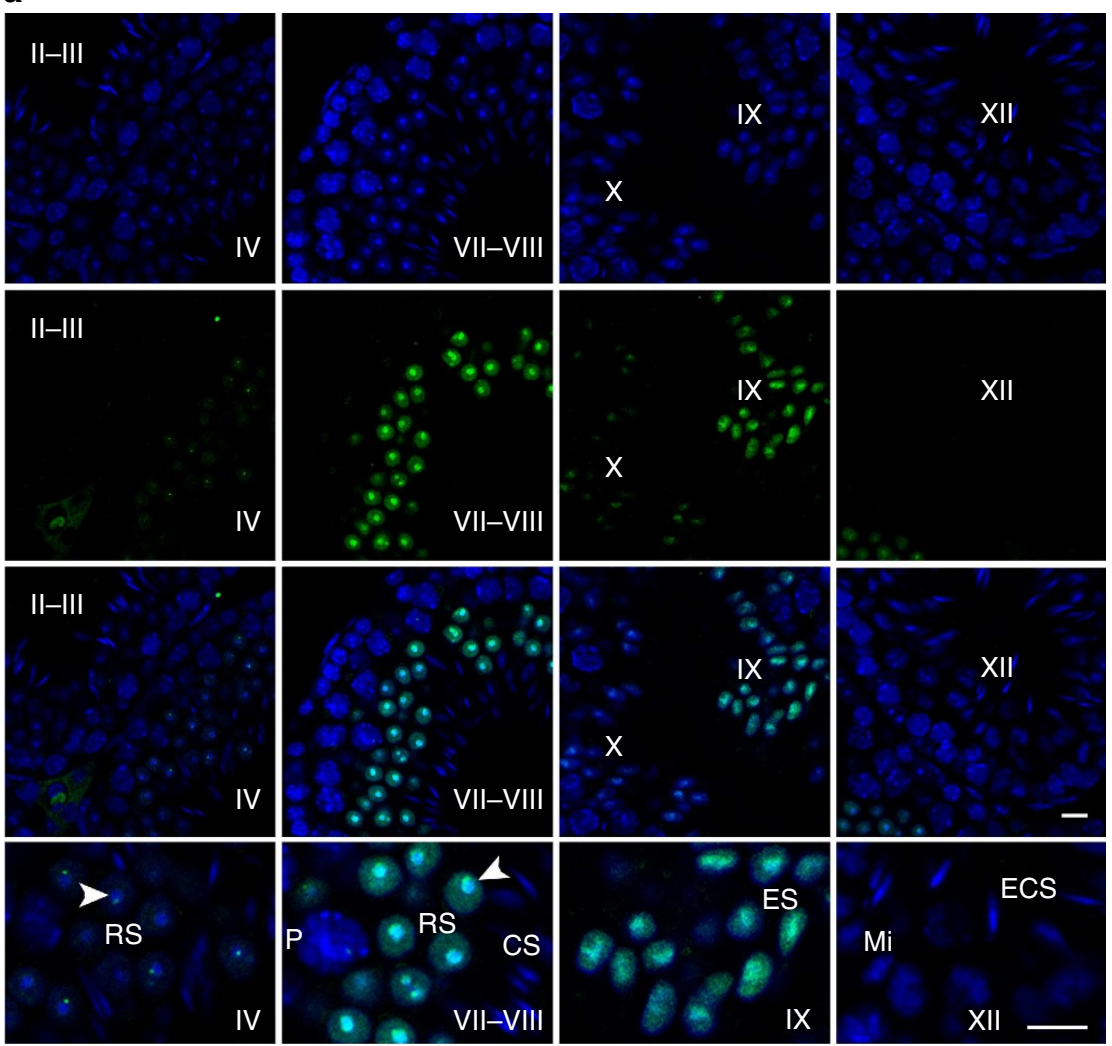

b
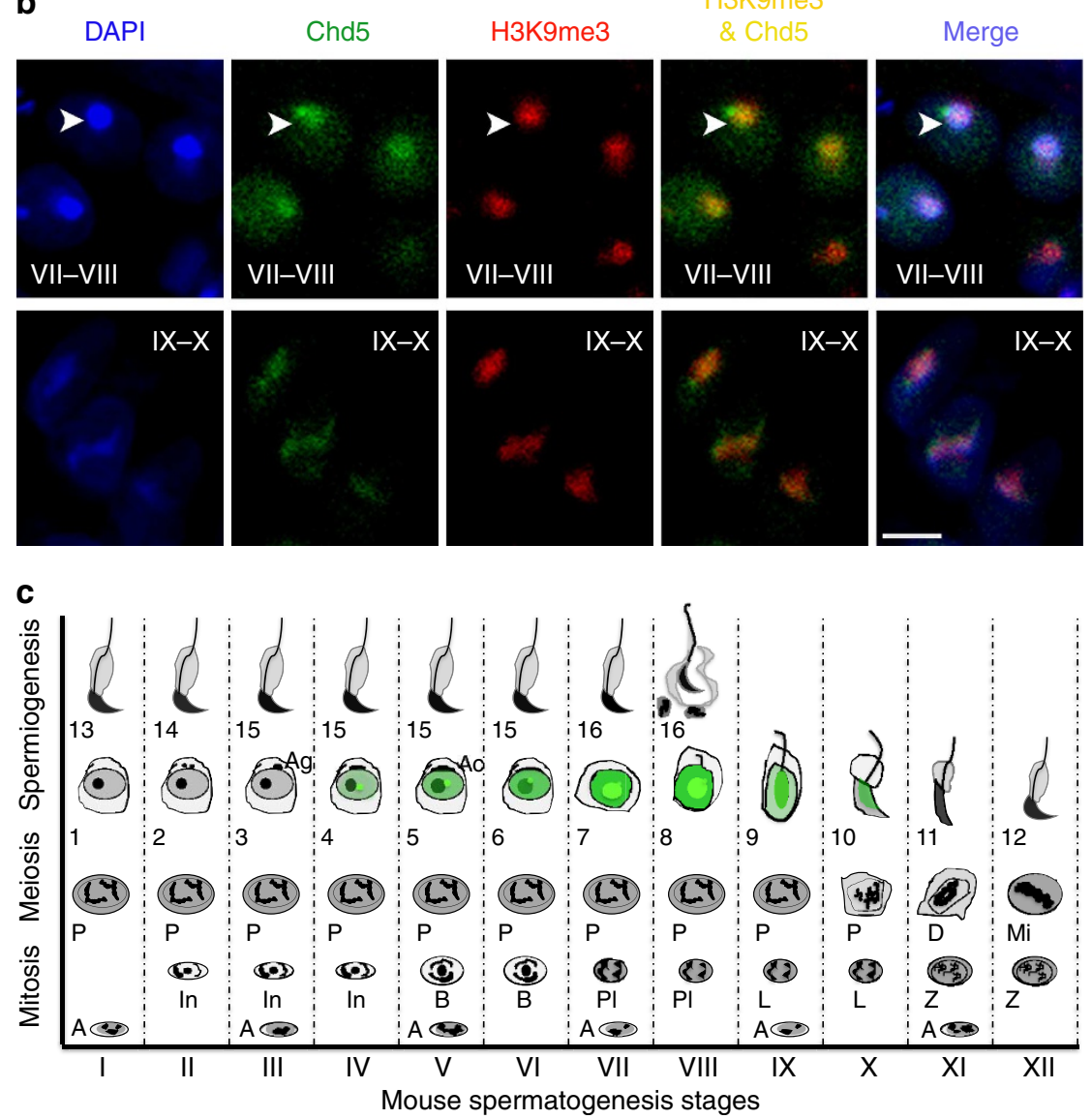
a

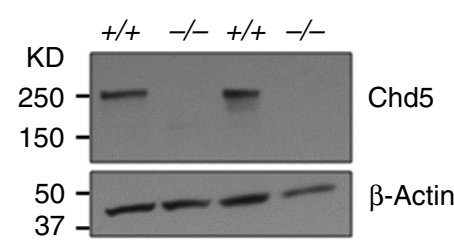

C

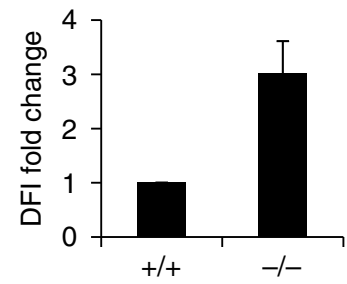

d

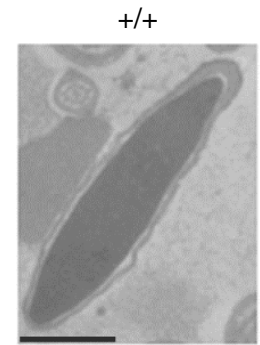

e

$+/+$

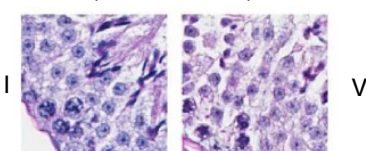

cosoly
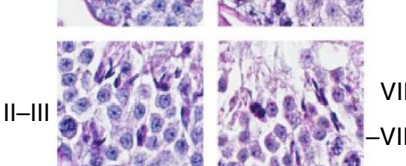

(1)
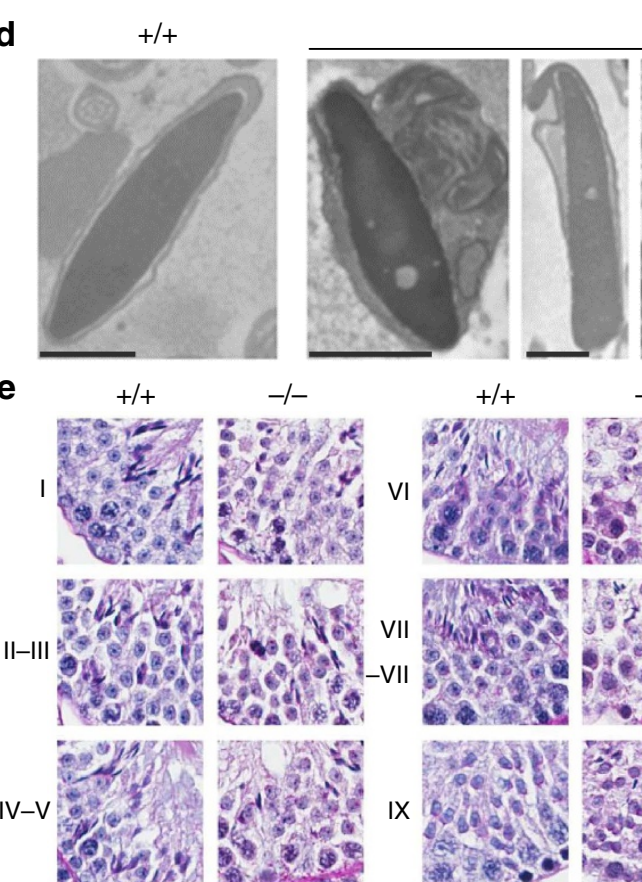

b $+/+$
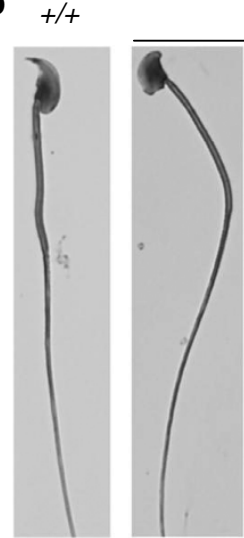

$-1-$

$-1-$

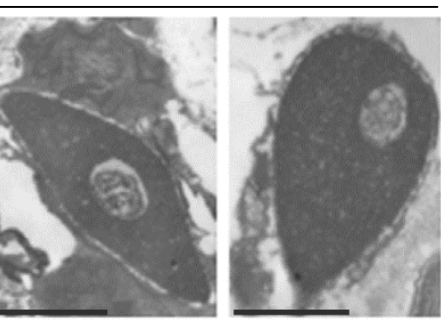

$+/+$

$-1-$

$+/+$

$-1-$

$\mathrm{VI}$
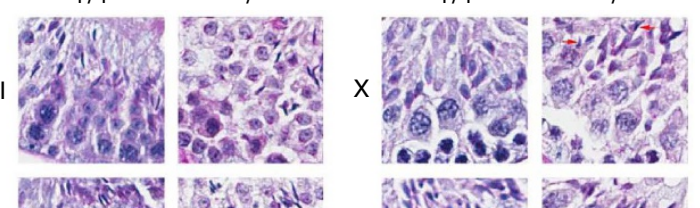

4Hazes $\%$

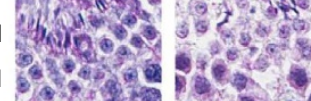

$X I$
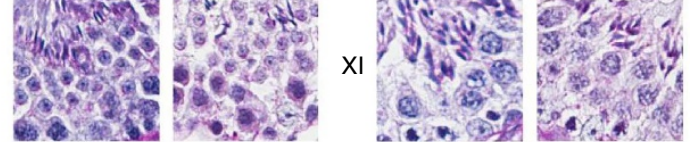

a $\rightarrow 2$

2.). 20.35

IX
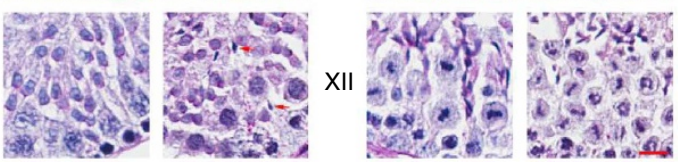

f

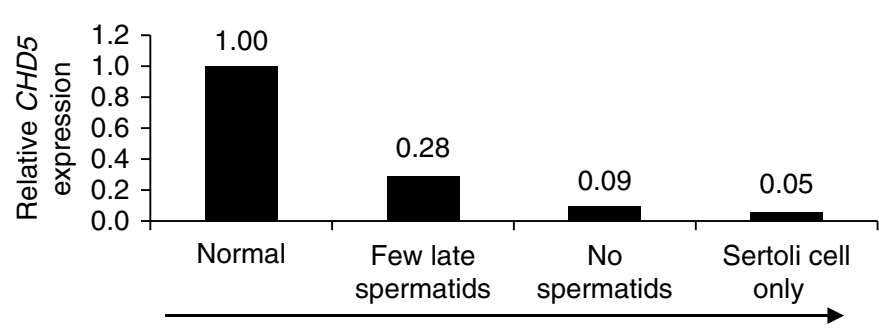

Human testis pathology

Figure 2 | Chd5 deficiency leads to defective spermatogenesis and chromatin condensation. (a) Western blot analyses indicates that Chd5 protein is not detectable in Chd5 Aam1-/- $(-/-)$ testis. A validated antibody ${ }^{10}$ raised against amino acids 1,524-1,705 of mouse Chd5 (which is not disrupted by gene targeting), was used for western blotting. $\beta$-Actin serves as a loading control. (b) Representative abnormal head morphology of Chd5 $5^{\text {Aam1 } 1-/-}$ sperm. Scale bar, $20 \mu \mathrm{m}$. (c) SCSA revealed impaired chromatin integrity of Chd5 Aam1-/- sperm. DFI, DNA Fragmentation Index (see Methods). Data are presented as mean \pm s.d. from four independent experiments. (d) Transmission electron microscopy analyses of sperm from Chd5 Aam1 $+/+$ and Chd5 $5^{\text {Aam } 1-/-}$ caudal epididymi. Chromatin is homogenously condensed in Chd5 ${ }^{A a m 1+/+}$ sperm, but appears loose and uneven with fibrillar texture and contains abnormal vacuoles in Chd5 ${ }^{\text {Aam1 } 1-/-}$ sperm nuclei. Scale bar, $1 \mu \mathrm{m}$. (e) Staged comparison of periodic acid-Schiff (PAS)-stained Chd5 ${ }^{\text {Aam1 } 1+/+}$ and Chd5 $5^{\text {Aam1 } 1-/-}$ testes. Roman numerals indicate the stages of the seminiferous tubules. A decrease in the number of elongated spermatids, especially at stages VII and VIII, is evident in Chd5 $5^{\text {Aam } 1-/ ~-~ t u b u l e s . ~ A r r o w s ~ i n ~ s t a g e ~ I X ~ a n d ~ X ~ m a r k ~ a b n o r m a l ~ r e t e n t i o n ~ o f ~ c o n d e n s e d ~ s p e r m a t i d s . ~ S c a l e ~ b a r, ~} 10 \mu \mathrm{m}$. (f) Relative CHD5 expression in human testis with normal versus clinically defined abnormal spermatogenesis. Data are derived from published microarray data set (ArrayExpress: E-TABM-234) of 39 human testis biopsy samples from 29 men with highly defined testicular pathologies and 10 men with normal spermatogenesis. RNA was prepared from the testis biopsies and analysed for gene expression using Affymetrix GeneChip. Data were analysed through NextBio ${ }^{18}$. Arrow indicates increased severity of spermatogenic defect. 


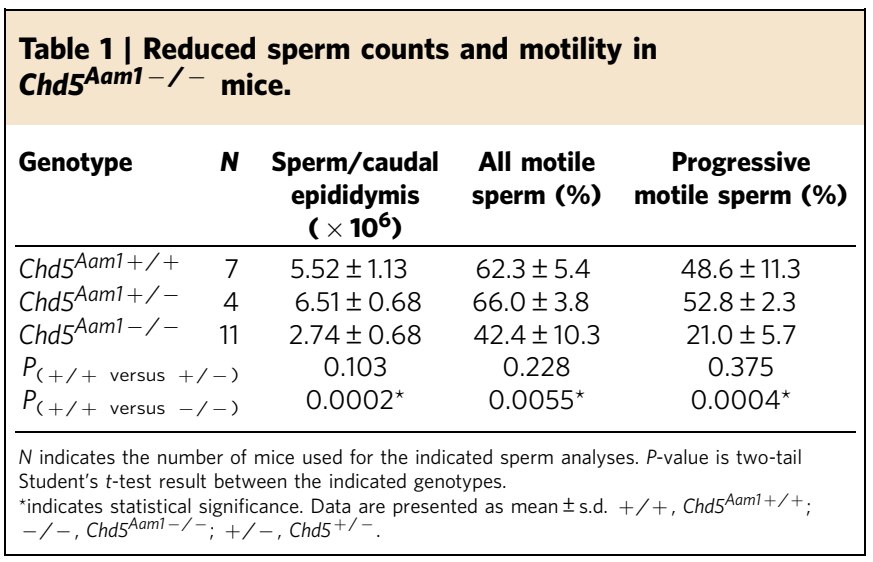

the sperm that were produced had compromised motility and a higher proportion of morphological abnormalities (Table 1, Fig. 2b and Supplementary Table 2). Using in vitro fertilization (IVF), we found that Chd5 $5^{\text {Aam1-/- }}$ sperm failed to fertilize wild-type oocytes (Supplementary Table 3). Although it might be expected that some functional sperm from sub-fertile Chd5 $5^{\text {Aam 1- / }-}$ mice should be able to fertilize oocytes, none of the sperm obtained from three different Chd5 $5^{\text {Aam } 1-I-}$ mice were able to generate blastocysts in vitro. This may be because each of the three males tested happened to be sterile rather than sub-fertile, or that IVF conditions compromised the sperm that would have been able to fertilize oocytes under in vivo conditions. A possible explanation for the range of severity of the fertility phenotype in different mice was genetic background, as 129Sv embryonic stem cells were used to generate the Chd5 $5^{\text {Aaml } 1}$ allele, with mice being backcrossed for over four generations onto the C57BL/6 background before heterozygotes were intercrossed. To determine whether genetic background affected fertility, we established a second Chd5deficient mouse model (carrying the Chd5 $5^{\operatorname{Tm} 1 b}$ null allele ${ }^{15}$ ) that was in a $100 \%$ pure $\mathrm{C} 57 \mathrm{BL} / 6$ genetic background, and assessed male fertility (Supplementary Fig. 5, Supplementary Table 4). We found that Chd5 $5^{\operatorname{Tm} 1 b-/-}$ male mice were also either sterile or sub-fertile. Another Chd5-deficient mouse model in a pure 129E background also showed that homozygous male mice exhibited variable pathology ranging from absence of sperm to near-normal sperm count, although six homozygous males tested did not produce any progeny over the 2 -month period analysed ${ }^{16}$. These data suggest that Chd5 deficiency compromises sperm production and male fertility and that this phenotype exhibits inherent variability in different individuals.

To assess chromatin integrity in sperm from Chd5-deficient mice, we used the sperm chromatin structure assay (SCSA) (Fig. 2c). SCSA revealed that DNA fragmentation was enhanced in Chd5 $5^{\text {Aam } 1-/-}$ sperm, reflecting a compromise in chromatin integrity ${ }^{17}$. Consistent with this finding, transmission electron microscopy showed that whereas chromatin within nuclei of wild-type sperm is homogeneously condensed, less-condensed chromatin with a punctate texture and uneven density, as well as the presence of abnormal vacuoles, were observed in nuclei of Chd5 $5^{\text {Aam 1-/- }}$ sperm (Fig. 2d). These findings show that Chd5 deficiency leads to defective chromatin compaction in sperm.

Since compromised fertility can be caused by a deregulation of sex hormones, we investigated this possibility (Supplementary Fig. 6). However, we did not detect a significant alteration in sex hormones in Chd5 $5^{\text {Aam 1-l- }}$ male mice relative to controls. Histological analyses of testes revealed that seminiferous tubules of Chd5 $5^{\text {Aam 1-l- }}$ mice contained fewer elongated spermatids relative to controls, with an abnormal retention of condensed spermatids within stage IX and X tubules (Fig. 2e, Supplementary
Fig. 7). The extent of histological abnormalities varied among individual Chd5 $5^{\text {Aam1-1- }}$ mice (Supplementary Fig. 7), in agreement with the variable severity of infertility observed in male Chd5-deficient mice. In contrast to post-meiotic defects, we did not observe differences in spermatogenic cells (spermatogonia, spermatocytes, round spermatids) or in somatic cells (Sertoli cells, Leydig cells) in Chd5 $5^{\text {Aam1-/ - }}$ testes (Fig. 2e, Supplementary Fig. 7). These findings indicate that Chd5 deficiency disrupts the elongation and condensation steps of post-meiotic sperm maturation, consistent with Chd5's peak of expression in step 7-8 round spermatids, the phase immediately preceeding extensive chromatin remodelling.

To determine whether our findings from Chd5-deficient mice might be relevant to human cases of male infertility, we analysed a previously established gene expression data set of testes biopsies from 39 men (29 men with highly defined testicular pathology and 10 men with normal spermatogenesis) ${ }^{18}$. This analysis revealed that men with spermatogenic defects had lower CHD5 expression relative to controls and that the clinical grade of spermatogenic defect correlated inversely with $\mathrm{CHD} 5$ expression (Fig. 2f). While this is a correlation rather than evidence for causality, it suggests that future efforts should be made to determine whether compromised CHD5 contributes to male infertility in humans.

Chd5 deficiency disrupts histone-to-protamine replacement. During mammalian spermiogenesis, the majority of canonical histones are removed and replaced by histone variants and transition proteins, which are subsequently replaced by protamines $6,7,19,20$. This histone-to-protamine replacement process repackages the sperm genome at least sixfold more compact than its somatic counterpart ${ }^{8}$. To define the mechanism whereby Chd5 deficiency compromises chromatin compaction during spermiogenesis, we used western blotting to assess expression of a panel of somatic histones, transition proteins and protamines in elutriation-purified spermatids at different steps of spermiogenesis (Supplementary Fig. 8). The core nucleosomal histones $(\mathrm{H} 1, \mathrm{H} 2 \mathrm{~A}, \mathrm{H} 2 \mathrm{~B}, \mathrm{H} 3$ and $\mathrm{H} 4)$ were quickly depleted after the round spermatid steps in wild-type testes, with minimal retention in condensing and condensed spermatids (Fig. 3a). In contrast, these core histones were retained to a higher extent in Chd5 $5^{\text {Aam } 1-1-}$ differentiated spermatids, which implied ineffective histone removal. In addition, transition proteins (Tnp1 and Tnp2) and protamines (Prm1 and Prm2) had elevated expression in differentiated Chd5 $5^{\text {Aam 1-/- }}$ spermatids (Fig. 3b,c), likely contributing to impaired fertility of Chd5 $5^{\text {Aam 1 - I- }}$ mice, as precise control of levels of Prm1 and Prm2 are critical for male fertility ${ }^{21,22}$. Prm2 is first translated as a precursor protein that is subsequently processed into mature Prm2 through a multistep proteolytic cleavage. In Chd5 Aam1-/spermatids, there was enhanced expression of the Prm 2 precursor and partially processed Prm2 was present, indicating a defect in Prm2 processing, which may be because the overproduction of

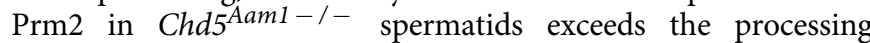
capacity of the cells. In addition, both Tnp1 and Tnp2 deficiency have been shown to increase levels of the Prm2 precursor and partially processed forms of Prm2 (refs 23,24). Thus, the abnormal elevation of Tnp1 and Tnp2 in Chd5 Aam1-1- spermatids may also contribute to defective Prm2 processing. Consistent with the observation in spermatids, western blotting of lysates from mature sperm also revealed enhanced retention of core nucleosomal histones and elevated Prm1 and Prm2 expression in Chd5 $5^{\text {Aam1-l- }}$ sperm (Fig. 3d). Furthermore, immunofluorescent analyses of testes showed enhanced expression of Tnp1, Tnp2, Prm1 and Prm2 in 

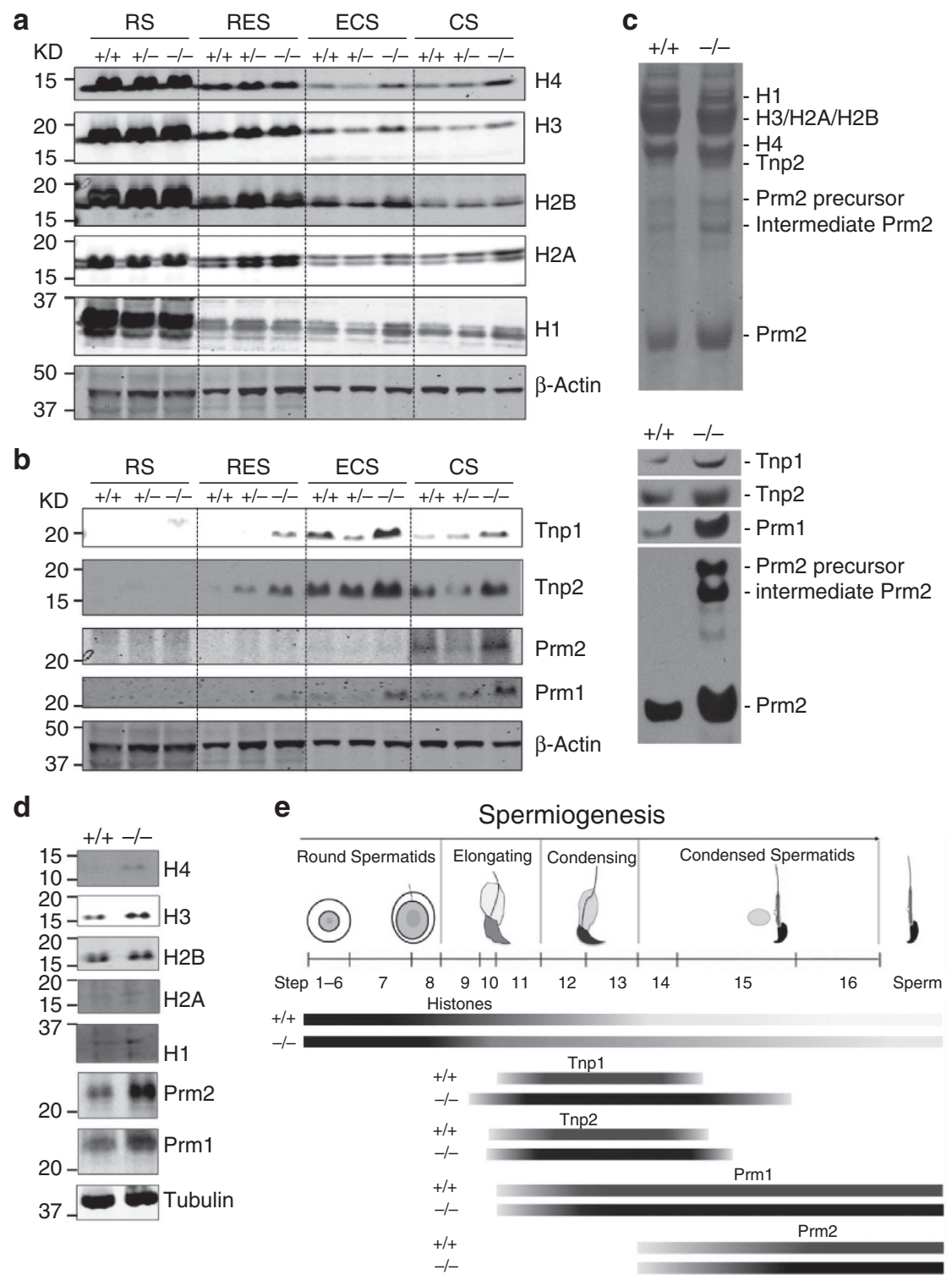

Figure 3 | Chd5 modulates histone removal and homeostasis of transition proteins and protamines. (a,b) Western blot analyses of protein lysates from purified spermatids at different spermiogenic stages. RS, round spermatids; RES, round and early elongating spermatids; ECS, elongating and condensing spermatids; CS, condensed spermatids. Increased retention of histones ( $\mathrm{H} 4, \mathrm{H} 3, \mathrm{H} 2 \mathrm{~B}, \mathrm{H} 2 \mathrm{~A}$ and $\mathrm{H} 1$ ) as well as elevated transition proteins (Tnp1, Tnp2) and protamines (Prm1, Prm2) are observed in elongating, condensing and condensed spermatids, but not in round spermatids of Chd5 $5^{\text {Aam1- / - }(-/-) ~ t e s t i s . ~} \beta$-Actin serves as a loading control. Levels of histones, transition proteins and protamines in Chd5 ${ }^{\text {Aam } 1+/-}$ spermatids are in general similar to the levels in Chd5 ${ }^{A a m 1}+/+$ counterparts, with some variability among different Chd5Aam1+/- samples. (c) Top panel, Coomassie blue staining of basic protein extracts from Chd5 $5^{A a m 1+/+}$ and Chd5 $5^{\text {Aam } 1-/-}$ sonication-resistant spermatids (SRS) separated using urea-acid gel electrophoresis. Equal amounts of proteins are loaded. Bottom panel, western blot analyses of the same basic protein extracts show an increase in Tnp1, Tnp2, Prm1 and Prm2 in Chd5Aam1-/- SRS. The increase in the Prm2 precursor and intermediate Prm2 indicate deficient processing of the Prm2 precursor

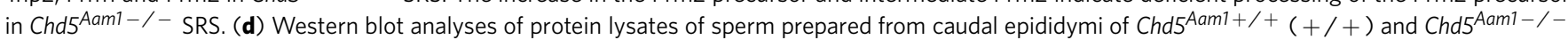
$(-/-)$ mice reveal increased retention of nucleosomal histones and elevated Prm1 and Prm2 in Chd5 $5^{\text {Aam } 1-/-}$ sperm. $\mathrm{H} 4, \mathrm{H} 2 \mathrm{~A}$ and $\mathrm{H} 1$ are barely detectable in Chd5 $5^{A a m 1}+/+$ sperm, but are detected in Chd5 Aam1 - / - sperm. H3 and H2B are detectable in both Chd5 Aam1 $+/+$ and Chd5 $5^{A a m 1-/-}$ sperm, but are higher in Chd5 Aam1 $-/-$ sperm. Tubulin serves as a loading control. (e) Illustration of the dynamics of histones, transition proteins and protamines during spermiogenesis in Chd5Aam1 $+/+(+/+)$ and Chd5Aam1- $/-(-/-)$ testes. For each nuclear protein, the darker colour of the bar in Chd5 ${ }^{\text {Aam } 1-/-}$ testis indicates stronger expression of the protein at the indicated spermatogenic steps relative to Chd5 $5^{\text {Aam } 1+/+}$ counterparts.

Chd5 $5^{\text {Aam1 - / - }}$ spermatids (Fig. 3e, Supplementary Figs 9-12). In addition, Tnp1 was precociously expressed in step 9-10 spermatids and had extended expression until step 15 spermatids in Chd5 $5^{\text {Aam } 1-/-}$ testes. These findings indicate that Chd5 deficiency perturbs the histone-to-protamine transition that occurs during spermiogenesis, leading to aberrant retention of nucleosomal histones and elevated levels of transition proteins and protamines.

Chd5 mediates histone removal and DNA repair. To assess the mechanism of how Chd5 deficiency leads to aberrant histone 
removal we focused on $\mathrm{H} 4$ hyperacetylation, a molecular event occurring in early elongating spermatids that is essential for histone-to-protamine replacement in Drosophila ${ }^{25}$, and considered the same for spermiogenesis in mammals ${ }^{6,25,26}$. Using immunofluorescence, we determined that Chd5 had similar expression dynamics as $\mathrm{H} 4$ hyperacetylation during spermiogenesis, but immediately preceded it (Fig. 4a). In step 9-10 spermatids, Chd5 colocalized with $\mathrm{H} 4$ acetylation, suggesting that Chd5 modulates $\mathrm{H} 4$ hyperacetylation during spermiogenesis (Fig. 4a). Consistent with this hypothesis, immunofluorescent analyses revealed that $\mathrm{H} 4$ acetylation was compromised in Chd5 Aam1-/- spermatids (Fig. 4b). In Chd5 $5^{\text {Aam } 1+/+}$ testis, $\mathrm{H} 4$ hyperacetylation was detected in step 9 spermatids and showed peak expression in step 10-12. H4 acetylation decreased in step 13 spermatids, and was not detectable at later steps. Whereas $\mathrm{H} 4$ acetylation was detected within step 9-13 spermatids of Chd5 $5^{\text {Aam1-/- }}$ testis, its expression was substantially reduced in Chd5 $5^{\text {Aam } 1+1+}$ a
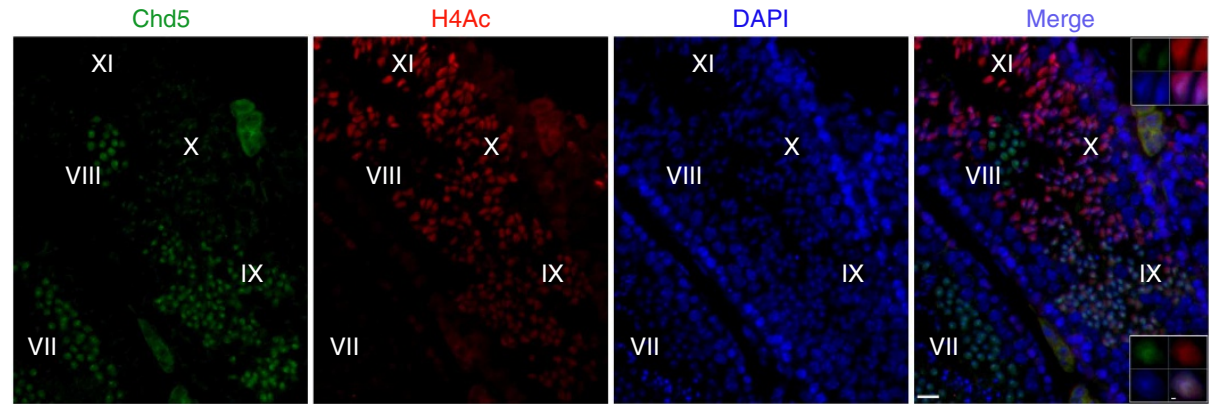

b
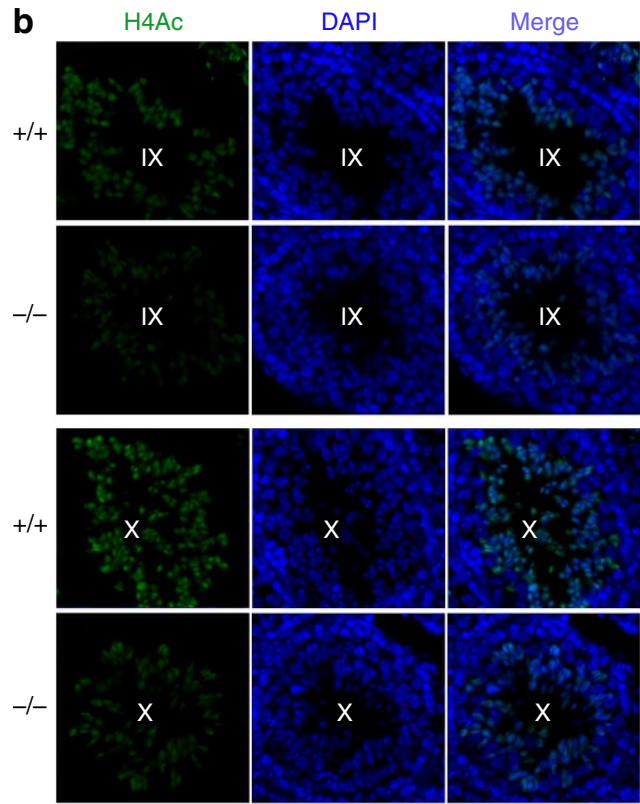

C
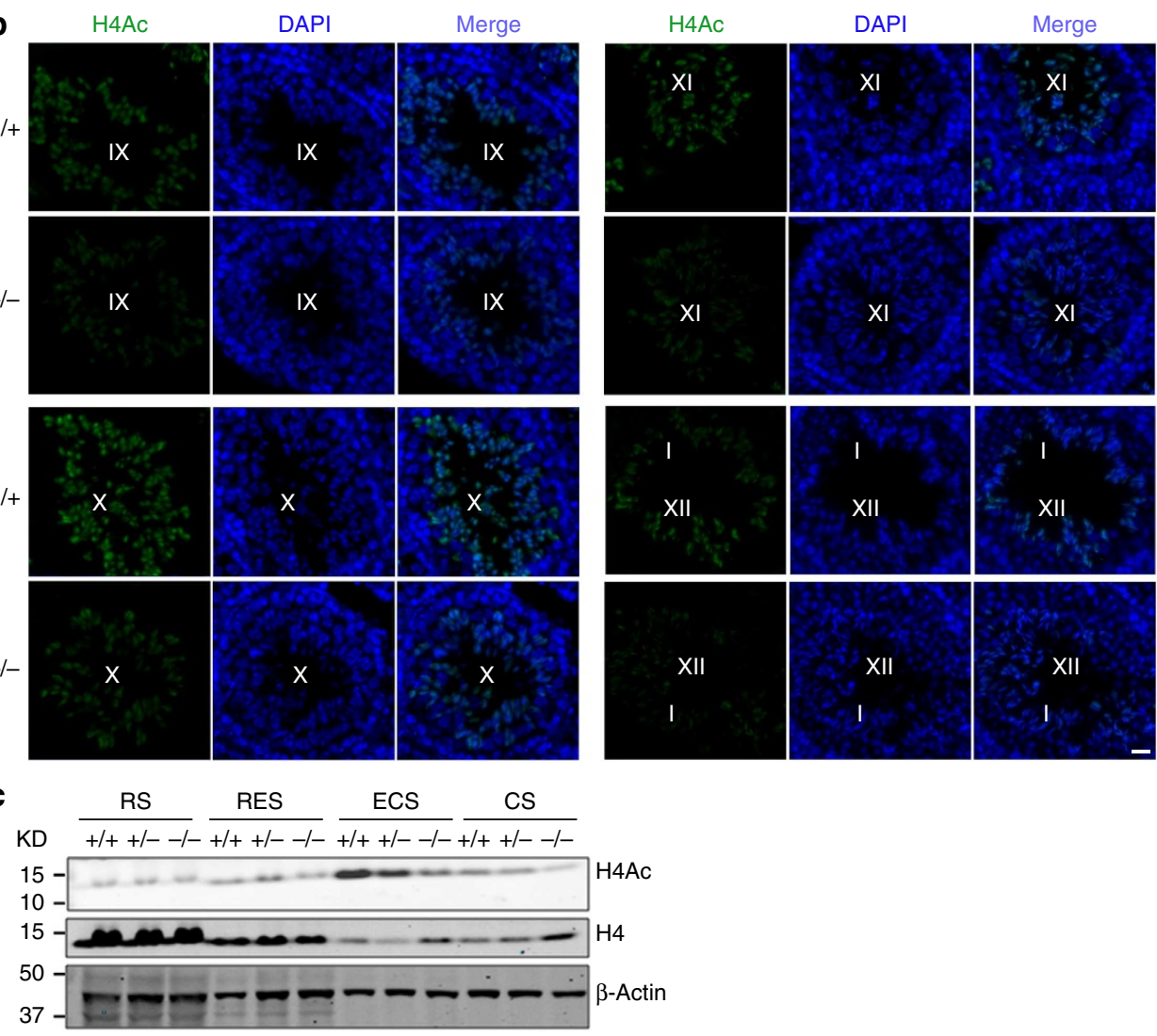

Figure 4 | Chd5 deficiency leads to compromised H4 acetylation during spermiogenesis. (a) Co-staining of Chd5 and H4 acetylation (H4Ac) in wild type testes. Roman numerals indicate spermatogenic stages of the marked tubular areas. Top and bottom inserts show higher magnification view of step 10 and step 9 spermatids, respectively. Both Chd5 and H4Ac are enriched in DAPI-intense regions within nuclei of step 9 and 10 spermatids in wild-type testes. H4Ac is detected by a mouse monoclonal antibody against pan-H4K5/8/12/16Ac. Scale bars, $20 \mu \mathrm{m}$ in main panels and $1 \mu \mathrm{m}$ for inserts.

(b) Immunofluorescence analyses of $\mathrm{H} 4$ acetylation in $C h d 5^{A a m 1}+/+(+/+)$ and $C h d 5^{A a m 1-} /-(-/-)$ seminiferous tubules. Roman numerals indicate spermatogenic stages of the marked tubules. $\mathrm{H} 4$ hyperacetylation starts at step 9 spermatids of stage IX tubules, exhibits peak expression from step 10 of stage $\mathrm{X}$ tubules to step 12 spermatids of stage $\mathrm{XII}$ tubules and decreases in step 13 spermatids in stage I tubules in Chd5 Aam $1+/+(+/+)$ testis. $\mathrm{H} 4$ acetylation is weaker from step 9 to 13 spermatids in Chd5 Aam1 $-/-(-/-)$ testis than in the Chd5 Aam1 $+/+$ counterparts. H4Ac was detected by a rabbit polyclonal antibody against pan-acetylation of $\mathrm{H} 4$. Scale bar, $20 \mu \mathrm{m}$. (c) Western blot analyses of purified spermatids at different spermiogenic

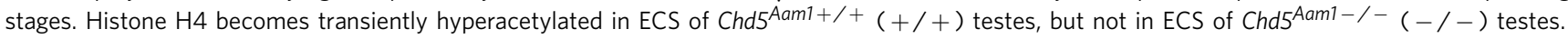
$\mathrm{H} 4 \mathrm{Ac}$ was detected by a rabbit polyclonal antibody against pan-acetylation of H4. $\beta$-Actin serves as a loading control. RS, round spermatids; RES, round and early elongating spermatids; ECS, elongating and condensing spermatids; CS, condensed spermatids. 
spermatids. Furthermore, western blotting of lysates from elutriation-purified spermatid fractions showed that whereas more total H4 was retained, H4Ac was severely compromised in differentiated Chd5 $5^{\text {Aam } 1-l-}$ spermatids (Fig. 4c). Consistent with the findings in Chd5 $5^{\text {Aam } 1-/-}$ mice, Chd5 $5^{\text {Tm } 1 b-/-}$ testes also showed compromised histone $\mathrm{H} 4$ acetylation (Supplementary Fig. 13). These findings indicate that acetylated $\mathrm{H} 4$ is compromised in Chd5-deficient testes.

Following H4 hyperacetylation, acetylated histone tails are recognized by Brdt, a testis-specific member of the BRD bromodomain-containing protein family that has been shown to induce eviction of nucleosomes ${ }^{6,27,28}$. However, how Brdt mediates nucleosome eviction is not clear. Likely, Brdt recruits chromatin remodelers to remodel and evict hyperacetylated nucleosomes. We therefore asked whether the compromised $\mathrm{H} 4$ acetylation and Chd5 deficiency perturbed nucleosome eviction in Chd5 $5^{\text {Aam1-1- }}$ spermatids. Using an antibody specific for intact nucleosomes ${ }^{29}$, we found that nucleosomes were detectable through step 11 of spermatid maturation in wild-type testes, but were depleted at later steps (Fig. 5a). However, we found that nucleosomes were aberrantly retained in Chd5 $5^{\text {Aam 1-/- }}$ spermatids as late as step 14 (Fig. 5b), indicating that nucleosomes were ineffectively evicted. Thus, both $\mathrm{H} 4$ acetylation and nucleosome eviction are compromised by Chd5 deficiency.
Nucleosome eviction generates DNA supercoiling tension that needs to be relieved. Previous studies indicate that topoisomerase II beta (Top2 $\beta$ ) catalyses resolution of such supercoils in elongating spermatids, during which double-strand breaks (DSBs) are generated. A DNA damage response is then triggered to repair the DSBs in order to maintain genome integrity ${ }^{30-33}$. We found that Chd5 expression is induced by DNA damage (Fig. 6a), suggesting that Chd5 plays a role in the DNA damage response as well. Indeed, TUNEL assays revealed an increase of DNA breaks in differentiated Chd5 $5^{\text {Aam } 1-/-}$ spermatids, most notably at steps $13-14$ (Fig. 6b), a time point when most DNA breaks are repaired in wild-type testes. In agreement with this finding, immunofluorescent analyses showed that whereas $\gamma$-H2A.X (a marker for the DSB-activated DNA damage response) is cleared in wild-type spermatids after step 12 , it is detected in Chd5 $5^{\text {Aam 1-l- }}$ spermatids as late as step 14 (Fig. 6c). Western blot analyses further confirmed an increase of $\gamma$-H2A.X in differentiated Chd5 $5^{\text {Aam } 1-/-}$ spermatids (Fig. 6d). Together, these findings indicate that Chd5 deficiency impairs H4 hyperacetylation, nucleosome eviction, and DNA damage repair during spermiogenesis.

Chd5 loss alters gene expression in spermatids. Tnp1, Tnp2, Prm1 and Prm2 are transcribed in round spermatids, but their

a
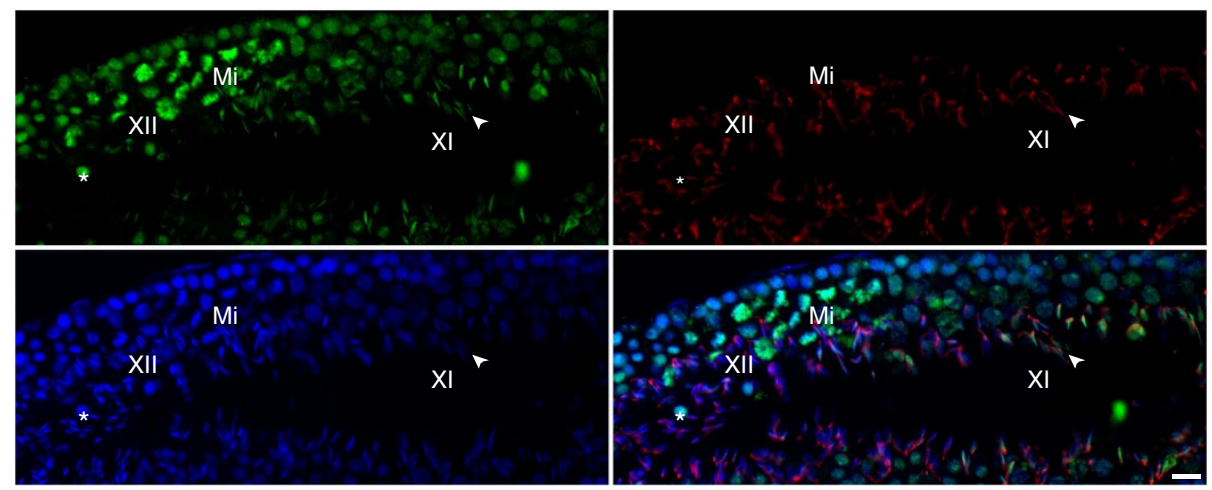

b Nucleosome

DAPI
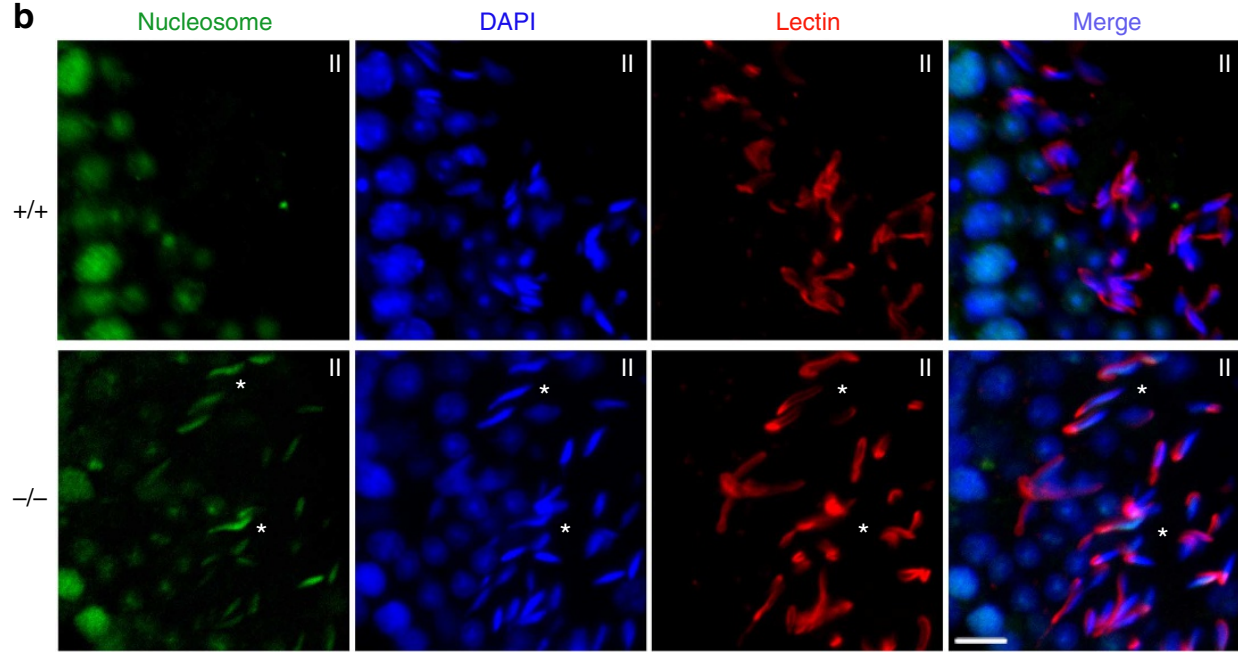

Figure 5 | Chd5 deficiency leads to inefficient nucleosome eviction during spermiogenesis. Roman numerals indicate the spermatogenic stages of tubular areas. (a) Nucleosomes are detected in step 11 spermatids (indicated by arrow head) of stage XI seminiferous tubules, but are depleted afterwards and not detectable in step 12 spermatids (indicated by *) of stage XII seminiferous tubule in wild-type testes. Green, nucleosomes; Red, lectin (visualizing acrosome for staging seminiferous tubules); Blue, DAPI; Mi, meiotic figure, a hallmark of stage XII tubules. (b) Immunostaining for nucleosomes shows that nucleosomes are retained in as late as step 14 spermatids of stage II seminiferous tubules in Chd5 Aam $1-/-(-/-)$ testes, while being absent in the Chd5 ${ }^{\text {Aam1 }+/+}(+/+)$ counterpart. Asterisks $\left(^{*}\right)$ mark nucleosome-positive condensing spermatids (step 14) in Chd5 ${ }^{\text {Aam1 } 1-/-}$ tubules. Scale bars, $10 \mu m$. 
a

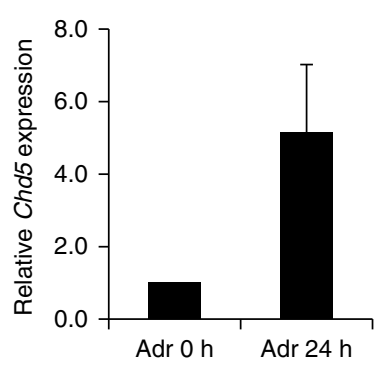

b

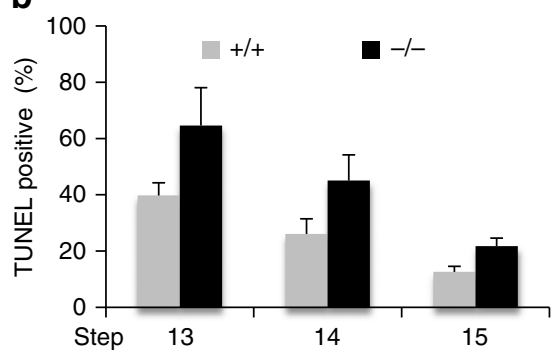

C

$\gamma-\mathrm{H} 2 \mathrm{~A} . \mathrm{X}$
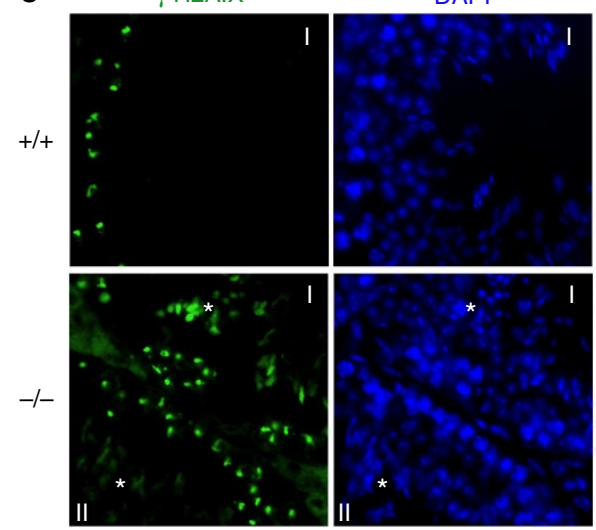

DAPI
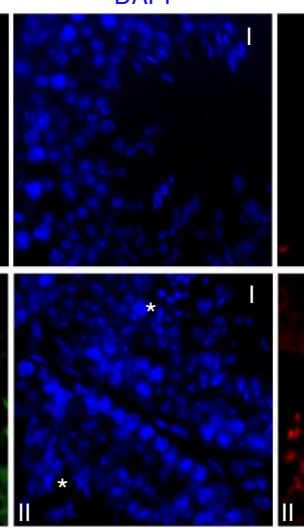
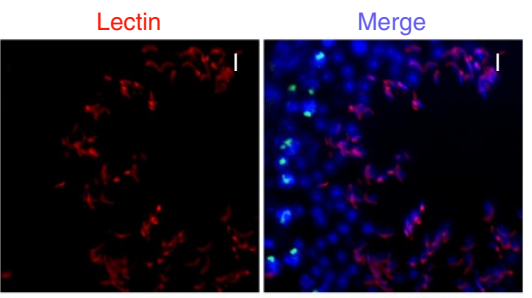

d

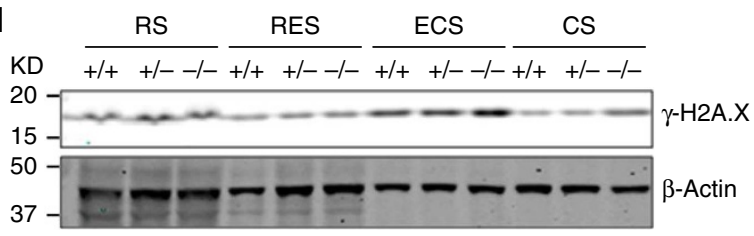

Figure 6 | Increased DNA damage in Chd5 $\mathbf{A a m 1}^{-/ \text {- }}$ spermatids. (a) qRT-PCR analyses shows that Chd5 expression is induced approximately fivefold when wild-type mouse embryonic fibroblasts are treated with the DNA-damaging agent adriamycin $\left(0.4 \mu \mathrm{g} \mathrm{ml}{ }^{-1}\right)$ for $24 \mathrm{~h}$. Results are normalized to $A c t b$ and the expression level at $\mathrm{O}$ is defined as 1. Data are presented as mean \pm s.d. from three independent experiments. Adr, adriamycin. (b) Increased TUNEL-positive condensing spermatids (steps 13-15) in Chd5 ${ }^{\text {Aam } 1-/-~ t e s t i s . ~ D a t a ~ a r e ~ p r e s e n t e d ~ a s ~ m e a n ~} \pm$ s.d. from three independent experiments. (c) Immunofluorescence analyses of $\gamma-H 2 A . X$ in Chd5 Aam1 $+/+(+/+)$ and Chd5 Aam1 $-/-(-/-)$ seminiferous tubules. Asterisks $\left({ }^{*}\right)$ mark $\gamma$-H2A.X-positive spermatids (step 13 in stage I and step 14 in stage II tubules) in Chd5 Aam1-/- testis. Scale bar, $20 \mu \mathrm{m}$. (d) Western blot analyses of purified spermatids at different spermiogenic stages show a transient upregulation of $\gamma-\mathrm{H} 2 \mathrm{~A}$.X in wild-type ECS, and increased accumulation of $\gamma$-H2A.X in Chd5 ${ }^{\text {Aam1- }-/-~ E C S ~ a n d ~ C S ~ c o m p a r e d ~ w i t h ~ w i l d-t y p e ~ c o u n t e r p a r t s . ~} \beta$-Actin serves as a loading control. RS, round spermatids; RES, round and early elongating spermatids; ECS, elongating and condensing spermatids; CS, condensed spermatids.

transcripts are stored in translationally repressed ribonucleoprotein particles before being later translated into protein within elongating spermatids ${ }^{34-36}$. To determine whether the increase in transition proteins and protamines within Chd5-deficient spermatids was due to enhanced expression at the transcript level, we used qRT-PCR to assess Tnp1, Tnp2, Prm1 and Prm2

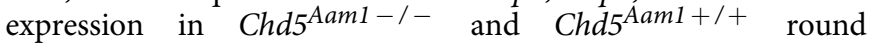
spermatids. Whereas only a slight increase of Tnp1, Tnp2 and Prm2 transcripts were detected, Prm1 transcript was increased $\sim 2.5$-fold in Chd5 $5^{\text {Aaml-l- }}$ round spermatids (Fig. 7a). Using chromatin immunoprecipitation-qPCR (ChIP-qPCR) analyses of nuclear lysates from testicular cells, we found an enrichment of Chd5 binding at the Prm1 promoter (region P: $-77 \mathrm{bp}$ to $+135 \mathrm{bp}$ ) (Fig. $7 \mathrm{~b}$ ). Less-pronounced Chd5 binding was observed at region $\mathrm{A}(-860$ to $-672 \mathrm{bp}), \mathrm{B}(-444 \mathrm{bp}$ to $-239 \mathrm{bp})$ and $\mathrm{C}(+397 \mathrm{bp}$ to $+585 \mathrm{bp})$, but not at region $5^{\prime}(-1,319 b p$ to $-1,164 \mathrm{bp})$. Collectively, these data suggest that Chd5 represses Prm1 transcription, whereas it negatively modulates expression of Tnp1, Tnp2 and Prm2 mainly post-transcriptionally.
As described above, a Chd5-intense focus near the edge of chromocentre in round spermatids showed proximity to the nucleolar marker fibrillarin (see Supplementary Fig. 3b), suggesting that Chd5 may play a role in rRNA biogenesis and translational control. qRT-PCR analyses of $45 \mathrm{~S}$ and $28 \mathrm{~S}$ rRNA expression revealed that $45 \mathrm{~S}$ rRNA was reduced by $\sim 30 \%$, and $28 \mathrm{~S}$ rRNA expression was reduced by $>50 \%$, in Chd5 $5^{\text {Aam1-l- }}$ round spermatids (Fig. 7c), indicating compromised rRNA biogenesis in Chd5 $5^{\text {AamP- }} /-$ spermatids. These results suggest a role of Chd5 in rRNA biogenesis during spermiogenesis, which is consistent with the known function of other $\mathrm{CHD}$ proteins such as $\mathrm{CHD} 4$ and $\mathrm{CHD} 7$ in positively regulating rRNA biogenesis 37,38 .

In addition to $\mathrm{H} 4$ hyperacetylation, replacement of canonical core nucleosomal histones with histone variants including those that are testis-specific is another key mechanism facilitating nucleosome destabilization and histone removal during spermatogenesis ${ }^{39-41}$. We examined expression of a panel of general and testis-specific histone variants in Chd5 $5^{\text {Aam1 } 1} /+$ and Chd5 $5^{\text {Aam 1-I- }}$ round spermatids via $\mathrm{qRT}-\mathrm{PCR}$, and discovered 
a
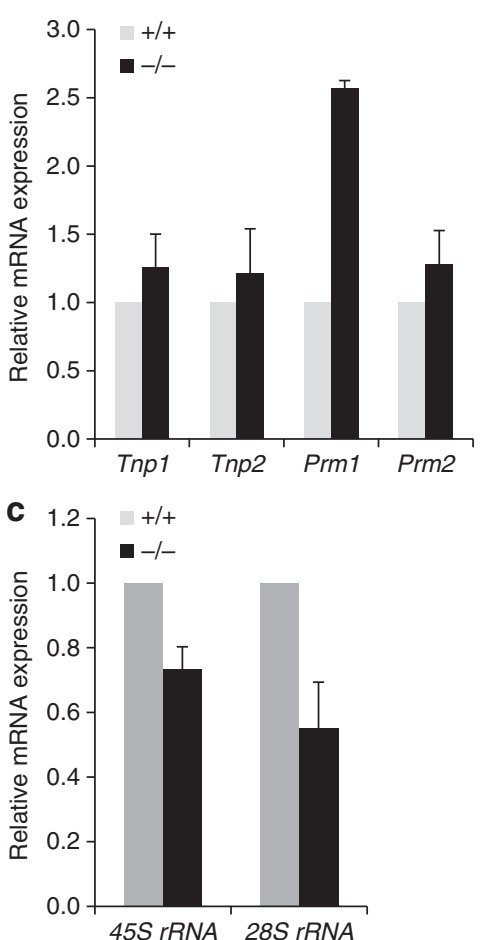
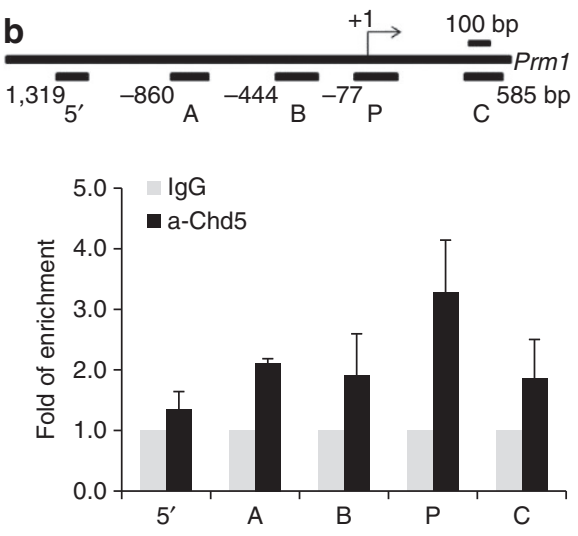

d

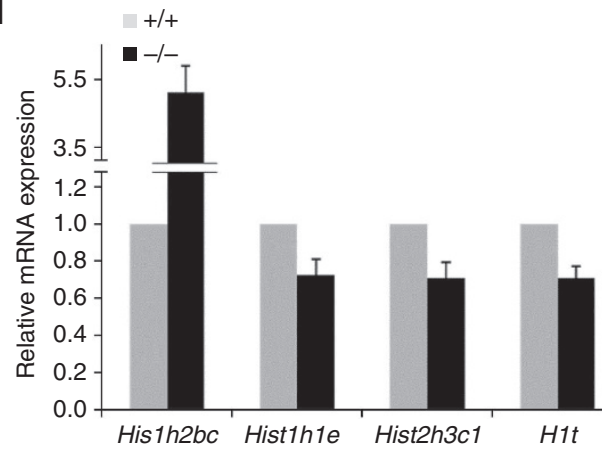

Figure 7 | Altered transcription of Prm1 and histone variants in Chd5-deficient spermatids. (a) qRT-PCR analyses of transition protein and protamine genes in Chd5 $5^{A a m 1+/+}$ and Chd5 ${ }^{A a m 1-/-}$ round spermatids. Results are normalized to Actb expression. Prm1 expression is increased by $\sim 2.5$-fold in Chd5 ${ }^{\text {Aam1 } 1 /-~ r o u n d ~ s p e r m a t i d s . ~ D a t a ~ a r e ~ p r e s e n t e d ~ a s ~ m e a n ~} \pm$ s.d. from four independent experiments. (b) Top panel, diagram of mouse Prm1 gene and location of primer sets used for ChIP-qPCR. Bottom panel, ChIP-qPCR analyses reveals enrichment of Chd5 at promoter region P ( -77 bp to $+135 \mathrm{bp}$ ) of Prm1 gene. The results are normalized to IgG control and are shown as fold of enrichment. Data are presented as mean \pm s.d. from four to five independent experiments. (c) qRT-PCR analyses of rRNAs shows compromised expression of $28 \mathrm{~S}$ and $45 \mathrm{~S}$ ribosomal RNAs in Chd5 Aam1-/$(-/-)$ round spermatids. Results are normalized to Actb expression. Data are presented as mean \pm s.d. from four to five independent experiments. (d) qRT-PCR analyses of histone variants revealed an approximately fivefold increase in expression of histone $\mathrm{H} 2 \mathrm{~B}$ variant Hist $7 \mathrm{~h} 2 \mathrm{bc}$ and modest decreases in expression of histone variants Histhhle, Hist2h3c1 and $H 1 t$ in Chd5Aam1-/- $(-/-)$ round spermatids. Results are normalized to Actb expression. Data are presented as mean \pm s.d. from three to five independent experiments.

that expression of the $\mathrm{H} 2 \mathrm{~B}$ variant Hist $1 \mathrm{~h} 2 b c$ was elevated over fivefold in Chd5 $5^{\text {Aam 1-/- }}$ round spermatids (Fig. 7d). Although little is known about the functions of Hist $1 h 2 b c$, its substantial increase in Chd5 $5^{\text {Aam 1-/ - }}$ round spermatids suggests that it might impact histone variant exchanges during spermiogenesis. In addition, an $\sim 30 \%$ decrease in expression of histone $\mathrm{H} 1$ variant Hist1hle, histone $\mathrm{H} 3$ variant $\mathrm{Hist} 2 \mathrm{~h} 3 \mathrm{cl}$ and testis-specific $\mathrm{H} 1$ variant $H 1 t$ were consistently observed in Chd5 $5^{\text {Aam } 1-/-}$ spermatids (Fig. 7d). These findings indicate that Chd5 deficiency alters transcript levels of specific histone variants during spermiogenesis.

In order to gain insight into the global gene expression changes resulting from Chd5 deficiency and to identify candidate Chd5 targets, we performed RNA sequencing (RNA-Seq) (Fig. 8). Chd5 is mainly expressed in round spermatids, where transcription is most active during spermiogenesis, with transcription globally being ceased afterwards. Using RNA-Seq, we compared mRNA expression profiles of round spermatids that had been elutriationpurified from five sets of Chd5 $5^{\text {Aam 1+/+ }}$, Chd5 $5^{\text {Aaml } 1+/-}$ and

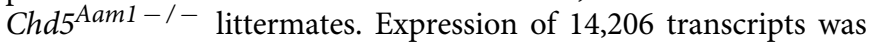
detected in at least one of the three genotypes. Two-hundred and sixty-one transcripts, or $1.8 \%$ of all the transcripts detected, showed a twofold or greater expression change in Chd5 $5^{\text {Aam1 - / - }}$ round spermatids compared with Chd5 $5^{\text {Aam } 1+/+}$ counterparts (false discovery rate $q=0.05$ ). Among the 261 transcripts, 156 transcripts or $59.8 \%$ were downregulated, whereas 105 transcripts, or $40.2 \%$, were upregulated in $C h d 5^{\text {Aam } 1-/-}$ round spermatids. Together, these results suggest that Chd5 deficiency leads to a rather limited alteration in global gene expression in round spermatids, and that Chd5 both activates and suppresses gene expression in round spermatids with a slight preference for activation. Gene ontology (GO) analysis of the gene expression changes showed clustering of GO terms including chromosome organization, response to DNA damage, acetylation, alternative splicing, nuclear export, protein transport, ubl (ubiquitin) conjugation, intracellular transport, endocytosis, cell cycle and MAPK pathway (Supplementary Fig. 14). Since Chd5 $5^{\text {Aam } 1+/-}$ male mice were fertile, we reasoned that genes that had expression changes only in Chd5 $5^{A m 1-/-}$ spermatids but not in Chd5 $5^{\text {Aam } 1+Y_{-}}$spermatids, or genes that had gradual expression changes dependent on Chd5 dosage, would be candidates most likely to contribute to the infertility of Chd5 $5^{\text {Aam 1-I- }}$ male mice. We thus further filtered the 261 transcripts through clustering with manual examination and identified a list of 155 transcripts that had expression changes only in Chd5 Aam1-/ - spermatids, or that had gradual expression changes that correlated with Chd5 dosage (Fig. 8a, Supplementary Fig. 15). Among the list, 90 transcripts were downregulated and 65 transcripts, including Hist $1 h 2 b c$, were upregulated in Chd5 ${ }^{\text {Aam } 1-7-}$ spermatids (Fig. 8a). qRT-PCR analyses for expression of 11 genes from this list, which included the positive control Hist $1 h 2 b c$ and represented a range of expression changes, 
a

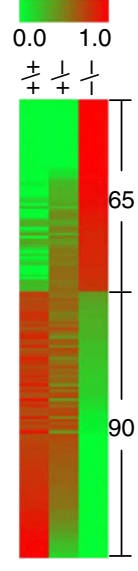

C

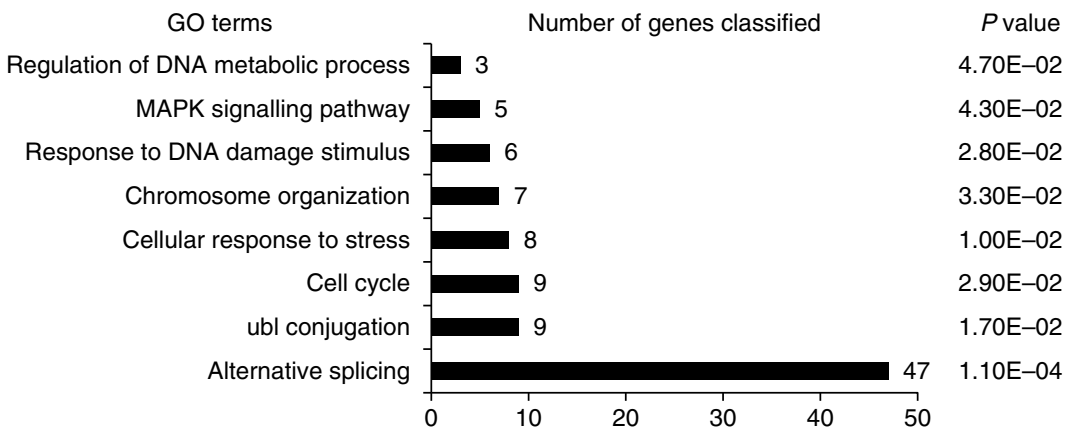

b

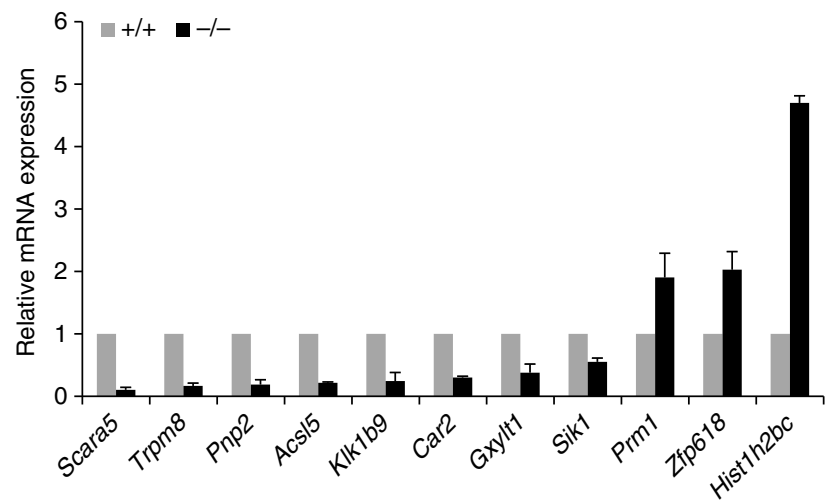

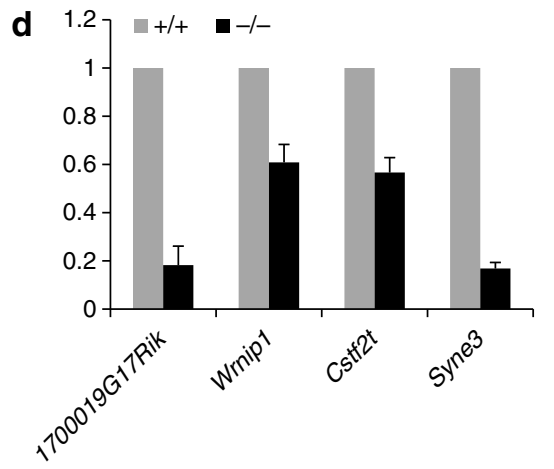

Figure 8 | RNA-Seq reveals global gene expression changes in Chd5 $\mathbf{A a m}^{-\boldsymbol{I}}$ - spermatids. (a) Heat map presentation of gene expression in

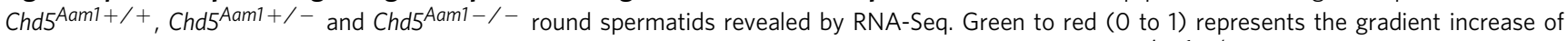
expression levels. Sixty-five transcripts show upregulation, and 90 transcripts show downregulation, in Chd5 $5^{\text {Aam1- } /-}$ round spermatids. (b) qRT-PCR validation of RNA-Seq revealed gene expression changes in round spermatids. Results are normalized to Actb expression and data are presented as mean \pm s.d. from four to five independent experiments. (c) GO analysis of the 155 transcripts that either show expression changes only in Chd5 $5^{A a m 1-/-}$ spermatids but not in Chd5 $5^{\text {Aam } 1+/-}$ spermatids, or that show gradual expression change from Chd5 $5^{\text {Aam } 1+/+}$ spermatids to Chd5 $5^{\text {Aam } 1+/-}$ spermatids to

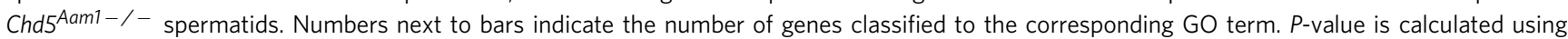
modified Fisher's exact test by DAVID $(\mathrm{v} 6.7)^{70}$. (d) qRT-PCR verification of compromised expression of genes implicated in histone acetylation, DNA damage response, RNA processing and nuclear integrity in Chd5 Aam1-/- spermatids. Results are normalized to Actb expression and Data are presented as mean \pm s.d. from four to five independent experiments.

was used to validate the expression changes revealed by RNA-Seq, attesting to the reliability of the RNA-Seq data and analyses (Fig. 8b). GO analysis of this list showed clustering of a subset of GO terms revealed from the original list, which include chromosome organization, response to DNA damage stimulus, alternative splicing, ubl conjugation, regulation of DNA metabolic process, cell cycle and MAPK signalling (Fig. 8c). A number of candidates implicated in acetylation (1700019G17Rik), DNA damage response (Wrnip1), RNA processing and translational control (Cstf2t) and nuclear structure maintenance (Syne3) were further verified using qRT-PCR (Fig. 8d, Supplementary Fig. 16). Altogether, these GO terms are consistent with the phenotypic impacts of Chd5 deficiency on chromatin compaction, DNA damage response and post-transcriptional modulation of transition proteins and protamines during spermiogenesis. Alterations in gene expression within these GO terms are likely contributing, at least partially, to the spermatogenic abnormalities of Chd5 $5^{\text {Aaml - / - male mice. }}$

\section{Discussion}

In this study, we show that Chd5 mediates a cascade of molecular events underlying histone removal during spermiogenesis including $\mathrm{H} 4$ hyperacetylation, histone variant expression, 
nucleosome eviction and DNA damage repair. Chd5 deficiency leads to disruption of these biological processes and increases histone retention in both spermatids and sperm. We reveal that Chd5 also modulates the homeostasis of transition proteins and protamines by suppressing expression of Prm1 transcriptionally and expression of Tnp1, Tnp2 and Prm2 post-transcriptionally. Chd5 deficiency results in elevated levels of both transition proteins and protamines. These findings unravel pleiotropic functions of Chd5 and highlight its multi-faceted role in orchestrating the extensive histone-to-protamine remodelling that occurs during male germ cell development.

Chd5 contains multiple domains (PHD domains, chromodomains, SNF2-like ATPase domain, DEAD/DEAH-box Helicase domain, SANT domain and DNA-binding motifs), which may enable its diverse functions during spermiogenesis. The SNF2-like ATPase domain defines the nucleosome remodelling function of CHD proteins ${ }^{42,43}$. Chd5 may facilitate nucleosome eviction during spermiogenesis through its ATPase domain, whose absence in Chd5-deficient spermatids would thus lead to inefficient nucleosome eviction. The ATPase domain is also implicated in DNA damage repair in somatic cells ${ }^{44}$ and may play a direct role in the DNA damage response during spermiogenesis and contribute to the increased DNA damage in Chd5-deficient spermatids. The DEAD/DEAH-box helicase domain may implicate Chd5 in RNA processing and the characteristic repression of mRNA translation in round spermatids, which could contribute to the abnormal post-transcriptional elevation of transition proteins and protamines in Chd5-deficient spermatids. It is known that the dual PHD domains of Chd5 preferentially bind $\mathrm{H} 3$ tails lacking $\mathrm{H} 3 \mathrm{~K} 4 \mathrm{me} 3$ in mouse embryonic fibroblasts ${ }^{10}$, whereas the chromodomains bind to H3K27me3 in neurons, both of which are important for Chd5 to mediate gene expression in somatic cells ${ }^{10,45}$. We also observe such patterns in mouse spermatids, as Chd5 is enriched in the chromocentre of spermatids, a heterochromatic region marked with H3K9me3 and H3K27me3, but lacking H3K4me3 (Fig. 1, Supplementary Fig. 2). This suggests that the PHD and/or chromodomains of Chd5 may also play important roles in regulating gene expression in spermatids, as RNA-Seq revealed that Chd5 deficiency leads to expression changes of specific gene sets. These multiple functional domains of Chd5 may work independently or in concert, enabling the diverse functions of Chd5 observed during spermiogenesis.

It is possible that there is a functional connection between the histone removal process and homeostasis of transition proteins and protamines. For example, transition proteins have been implicated in DNA repair during spermiogenesis ${ }^{46,47}$, thus the abnormal levels of transition proteins may contribute to the increased DNA damage in Chd5 $5^{\text {Aam } 1-1-}$ spermatids. On the other hand, deficient $\mathrm{H} 4$ acetylation may alter the chromatin structure of the Prm 1 locus, thereby contributing to its increased transcription, although it is counterintuitive that a decrease in $\mathrm{H} 4$ acetylation would result in transcriptional activation.

Chromatin remodellers are thought to be critical for the extensive chromatin remodelling taking place during the postmeiotic phase of spermiogenesis; however, little is known about their roles in this process. The chromatin remodeller Brg1 is essential for meiosis, and its deficiency leads to meiotic arrest with global alterations in histone modifications and chromatin structure in mice ${ }^{48}$. Acfl, which binds to chromatin remodeller Snf2h within the ACF complex, plays an essential role during post-meiotic spermiogenesis ${ }^{49}$. Deletion of Acf1 results in male infertility with increased DNA damage and spermiation defects, but without any detectable alterations in chromatin composition $^{49}$. Previous studies have also suggested roles of chromatin modifier Rnf8, a E3 ubiquitin ligase, in spermiogenesis. Lu et al. ${ }^{50}$ revealed that Rnf8 mediates $\mathrm{H} 2 \mathrm{~A}$ and $\mathrm{H} 2 \mathrm{~B}$ ubiquitination in elongating spermatids, and is critical for H4K16 acetylation and histone-to-protamine replacement. However, Sin et al..$^{51}$ later reported that Rnf8 deficiency does not affect H4K16 acetylation and histone-to-protamine exchange in spermatids, but instead compromises gene activation from inactive sex chromosomes in round spermatids. Thus, while a number of nuclear proteins have been implicated in spermatogenesis, Chd5, to our knowledge, is the first chromatin remodeller identified to play an orchestrating role in chromatin remodelling during post-meiotic spermiogenesis.

Consistent with Chd5 deficiency disrupting histone acetylation, DNA damage response and homeostasis of transition proteins and protamines during spermiogenesis, RNA-Seq reveals that Chd5 deficiency alters expression of genes encoding proteins underlying these processes, but does not cause a major change in global gene expression in round spermatids. We validate a number of candidate Chd5 target genes (for example, Cstf2t, 1700019G17Rik, Wrnip1 and Syne3) implicated in these processes. Notably, Cst $2 t$ encodes the RNA polyadenylation protein tauCstF-64, which is expressed during haploid spermatid differentiation ${ }^{52}$. Deletion of Cstf2t in mice disrupts postmeiotic development and leads to male infertility $y^{52}$. Similar to the heterogeneity of histopathology among Chd5 Aami-1- testes, Cstf2 $2 t^{-1-}$ male mice also display variable expressivity of sperm defects $^{52}$. These intriguing parallels suggest that deficient expression of Cstf2t in Chd5 ${ }^{\text {Aam } 1-/-}$ spermatids may contribute to the infertile phenotypes of Chd5 Aam 1-/- mice. In addition, the compromised expression of Cstf2t in Chd5 Aam1-l- spermatids may compromise polyadenylation and translational repression of transition protein and protamine mRNAs, thereby leading to an elevation in protein production, since translational repression of mRNAs in spermatids involves binding of protein repressors to poly (A) tails, and shortening of poly (A) tails of Tnp1, Tnp2, Prm1 and Prm2 mRNAs accompanies their translation activation ${ }^{53,54}$. 1700019G17Rik encodes a putative $\mathrm{N}$-acetyltransferase that is highly enriched in mouse testis but is absent in most other tissues (Supplementary Fig. 16a). We also found that 1700019G17Rik expression increases as round spermatids differentiate into elongating spermatids, the time point at which H4 hyperacetylation occurs (Supplementary Fig. 16b). These data suggest 1700019G17Rik as a potential acetyltransferase affecting $\mathrm{H} 4$ acetylation during spermiogenesis, and the $>80 \%$ decrease in expression may compromise $\mathrm{H} 4$ acetylation in Chd5 $5^{\text {Aam 1- /- }}$ spermatids. Wrnip1 stimulates the activity of DNA polymerase delta, rapidly accumulates at laserirradiated sites and is required for maintaining genome integrity ${ }^{55-57}$. Syne3 is a component of the nuclear envelope that tethers the nucleus to the cytoskeleton, and is critical for maintaining nuclear organization and structural integrity, as well as for development of the sperm head ${ }^{58-62}$. Compromised expression of Wrnip1 and Syne3 may contribute to the enhanced DNA damage and abnormal sperm head morphology, respectively, in Chd5 ${ }^{\text {Aam 1-1- }}$ testis. However, it is not yet clear whether these genes and other candidates revealed by RNA-Seq are direct Chd 5 target genes, a question that could be addressed by defining the global pattern of Chd5-bound loci in spermatids. A small proportion of histones and nucleosomes are retained in chromatin of wild-type sperm, preferentially at loci encoding proteins of developmental importance ${ }^{63}$. It would be interesting to know whether the aberrant histone retention in Chd5 $5^{\text {Aam P- / - }}$ sperm disrupts such a pattern, where the aberrantly retained histones locate in the genome of Chd5 $5^{\text {Aam } 1-/-}$ sperm, and to understand these implications for the developmental potency of the sperm. Future studies to characterize genome-wide distribution of Chd5, nucleosomal 
histones and specific histone modifications in Chd5-deficient spermatids and sperm should shed additional light on these questions.

We observed variation ranging from sterility to sub-fertility among individual Chd5 $5^{\text {Aam 1 - 9- }}$ male mice in a mixed C57BL/6 and $129 \mathrm{~S}$ background, as well as among individual Chd5 $5^{\mathrm{Tm} 1 \mathrm{~b}-/-}$ male mice in a pure C57BL/6 background. Such variability in infertility is similarly observed in Tnp1-1- and Tnp $2^{-/-}$male mice, as $\sim 40$ and $89 \%$ of $T n p 1^{-1-}$ and $T n p 2^{-1-}$ male mice, respectively, are sub-fertile ${ }^{23,24}$. The aberrant Tnp1 and Tnp2 levels in Chd5-deficient testes may contribute to the variable infertility among individual Chd5-deficient male mice. In addition, we observed increased DNA damage in Chd5deficient spermatids and sperm. The intrinsically variable extent of DNA damage may be more severe in testes of some Chd5deficient mice than in others, thus contributing to the variability of infertility.

$\mathrm{H} 4$ hyperacetylation in early elongating spermatids is shown to be essential for histone-to-protamine replacement in Drosophila ${ }^{25}$ and has been considered the same for mammalian spermiogenesis ${ }^{6,25,26}$. Our study shows that Chd5 deficiency leads to substantial deficiency in $\mathrm{H} 4$ acetylation in elongating spermatids and subsequent defects in nucleosome eviction and histone removal, supporting the notion that $\mathrm{H} 4$ hyperacetylation is indeed critical for efficient nucleosome eviction and histone removal during mouse spermiogenesis. However, most nucleosomes and histones eventually get removed in late Chd5 ${ }^{\text {Aam } 1-/-~ s p e r m a t i d s . ~ T h i s ~ s u g g e s t s ~ t h a t ~ w h e r e a s ~ H 4 ~}$ hyperacetylation is important for efficient nucleosome eviction and histone removal during mammalian spermiogenesis, it seems non-essential. Thus, whether $\mathrm{H} 4$ hyperacetylation is required for histone-to-protamine replacement during mammalian spermiogenesis warrants further investigation. In addition, the enzymes responsible for $\mathrm{H} 4$ hyperacetylation during spermiogenesis remain unidentified and are of great interest in the field. Our study identifies 1700019G17Rik as a candidate acetyltransferase for $\mathrm{H} 4$ hyperacetylation, providing a new promising target for future study.

The cascade of defects in $\mathrm{H} 4$ hyperacetylation, nucleosome eviction and DNA break repair during Chd5 ${ }^{\text {Aam 1-l- }}$ spermatid maturation provide functional evidence demonstrating the sequential order of these events, and suggest a model for the molecular events underlying the histone-to-protamine replacement process during spermiogenesis: H4 is first hyperacetylated, which along with other epigenetic modifications, leads to chromatin loosening and nucleosome eviction to facilitate histone removal and exposure of the DNA to allow for deposition of transition proteins and eventually protamines. DSBs are generated during nucleosome eviction to resolve supercoiling tension, and DNA damage response is activated to repair the DSBs, ensuring integrity of the sperm genome. This sequence of molecular events is in agreement with the model proposed by Leduc et al. ${ }^{30}$. Our findings establish functional evidence revealing the cascade of major molecular events underlying the histone-to-protamine replacement process, and provide a foundation to further elucidate this critical but elusive process.

\footnotetext{
Methods

Generation of Chd5-deficient mouse models. To generate the Chd $5^{\text {Aam } 1}$ mouse model, Chd5 locus was disrupted in AB2.2 ES cells using the MHPN20h05 MICER vector $^{64}$, and targeted ES cells were injected into C57BL/6 blastocysts through standard procedures. All animals were housed and utilized according to the Cold Spring Harbor Institutional Animal Care and Use Committee (IACUC) and the Association for Accreditation of Laboratory Animal Care International (AAALAC) policies. Progeny resulting from germline transmission were backcrossed to wildtype C57BL/6 mice, and Chd5 $5^{\text {Aam } 1+/-}$ mice were intercrossed to obtain homozygotes. To generate the Chd5 $5^{T m 1 b}$ mouse model, ES clones with
}

Chd5 ${ }^{T m}$ la(EUCOMM)Wtsi allele, which has exon 2 of Chd5 locus flanked by LoxP sites, were obtained from EUCOMM (European Conditional Mouse

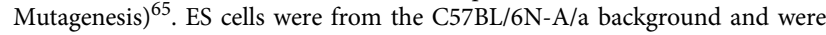
injected into albino B6 (C57BL/6J-Tyr c-2J) blastocysts through standard procedures. Progeny resulting from germline transmission, designated as Chd5 $5^{\text {Tmla } 1 /-}$ mice harboured a Chd5 allele with exon 2 flanked by LoxP sites. Chd5 Tm1a +/- mice were mated to CMV-Cre mice in the C57BL/6 background to obtain Chd5 $5^{T m 1 b+/-}$ mice (which had a Chd5 allele with exon 2 excised), and $C h d 5^{T m 1 b+/-}$ mice were intercrossed to obtain $C h d 5^{T m 1 b-/-}$ progeny.

Genotyping. For Southern blot genotyping of the Chd $5^{\text {Aaml } 1}$ model, the MHPN20h05 MICER vector was cut with AflII and the $2.6 \mathrm{~kb}$ excised fragment was gel-purified and used as a probe. Southern blotting of genomic DNA digested with BglII yielded the expected 7.8 and $10.4 \mathrm{~kb}$ endogenous and targeted alleles, respectively. Genotypes were differentiated based on dosage of the targeted allele (Chd5 $5^{\text {Aam1 1+/+ }}, 0$ copies; Chd5 $5^{\text {Aam1+/- }}, 1$ copy; Chd5 $5^{\text {Aam1-/- }}, 2$ copies). All genotypes had two copies of the endogenous band, which serve as an internal loading control and reference for dosage. For PCR-based genotyping, relative dosage of the neo-cassette in different genotypes $\left(\mathrm{Chd} 5^{\mathrm{Aam} 1+/+}, 0\right.$ copies;

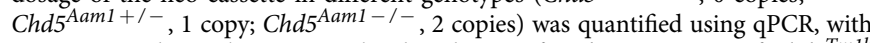
Neo-cassette dosage being normalized to dosage of Actb. Genotyping of Chd5 Tmlb mice was performed by PCR using primers (Supplementary Fig. 16) that amplify a 674-bp endogenous band specific for the wild-type Chd5 allele and a 456-bp targeted band specific for the targeted Chd5 $5^{T m 1 b}$ allele.

Antibodies. Antibodies used for western blot, immunoflurorescence and ChIP are as follows: anti-H3K27me3 (Cell Signaling no. 9756, 1:100 for IF, that is, immunofluorescence), anti-H2A (Cell Signaling no. 2578, 1:300 for WB, that is, Western blotting; for uncropped images, see Supplementary Fig. 17), anti-H4 (Cell Signaling no. 2935, 1:800 for WB), anti- $\gamma$-H2A.X (Cell Signaling no. 9718, 1:200 for IF; 1:1,500 for WB), anti-H3K9me3 (Active Motif no. 39385, 1:100 for IF), anti-H1 (Active Motif no. 39707, 1:800 for WB), anti-H2B (Active Motif no. 39125, 1:800 for WB), anti-H4Ac-pan (Active Motif no. 39243, 1:1,000 for WB; 1:200 for IF), anti-H3 (Abcam no. ab1791, 1:15,000 for WB), anti-Chd5 (Santa Cruz Biotechnology no. sc-68389, 1:1,500 for WB; 1:200 for IF), anti $\beta$-Actin (Sigma no. A2228, 1:2,000 for WB), anti-H4K5/8/12/14Ac (Millipore no. 05-1335, 1:100 for IF ), anti-lectin PNA Alex Fluor 568 (Invitrogen no. L32458, 1:4,000 for IF), antiPrm1 (Briar Patch Biosciences, Hup1N, 1:300 for WB; 1:150 for IF), antiPrm2 (Briar Patch Biosciences, Hup2B, 1:800 for WB; 1: 200 for IF), anti-Tnp1 (gift from Dr Stephen Kistler, University of South Carolina, 1:500 for WB; 1: 100 for IF ), anti-Tnp2 (gift from Dr Stephen Kistler, University of South Carolina, 1:1,000 for WB; 1: 200 for IF) and anti-nucleosome (mab no. 32, gift from Dr Jo H.M. Berden, Radboud University Nijmegen Medical Center, 1:300 for IF).

Histology and immunostaining. Testes were fixed in Bouin's fixative or $4 \%$ paraformaldehyde, embedded in paraffin and sectioned at $5 \mu \mathrm{m}$. Sections were deparaffinized in xylene and subjected to either PAS staining for histological analyses or immunofluorescent staining using the indicated antibodies.

Sperm counts and motility analysis. Individual caudal epididymi were minced in $200 \mu \mathrm{l} \mathrm{HTF}$ medium (Irvine Scientific). After $30 \mathrm{~min}$ incubation at $37^{\circ} \mathrm{C}$, the tissue pieces were separated from sperm by pipetting and passaging through a $70-\mu \mathrm{m}$ filter. Sperm counts and motility assessment were performed using the DRM-600 CELL-VU Sperm Counting Cytometer.

Sperm morphology. Air-dried smears were prepared from sperm suspended in PBS, stained with haematoxylin, and examined using light microscopy at $\times 100$ magnification. Head, neck and tail morphology was determined independently for each mouse, with separate counts of at least 100 cells per sample.

Sperm chromatin structure assay. SCSA was carried out as previously described $^{24}$ using a LSR II flow cytometer (Becton Dickinson). Briefly, a $0.2 \mathrm{ml}$ aliquot of sperm nuclei in TNE buffer (0.1 M Tris, $0.15 \mathrm{M} \mathrm{NaCl}$ and $1 \mathrm{mM}$ EDTA (pH 7.4)) was mixed with $0.4 \mathrm{ml}$ acid detergent solution $(0.15 \mathrm{M} \mathrm{NaCl}, 0.08 \mathrm{~N} \mathrm{HCl}$ and $0.1 \%$ Triton X-100, pH 1.4). After $30 \mathrm{~s}, 1.2 \mathrm{ml}$ acridine orange staining solution $\left(6 \mu \mathrm{g} \mathrm{ml}^{-1}\right.$ acridine orange, $0.1 \mathrm{M}$ citric acid, $0.2 \mathrm{M} \mathrm{Na} 2 \mathrm{HPO} 4,1 \mathrm{mM}$ EDTA and $0.15 \mathrm{M} \mathrm{NaCl}, \mathrm{pH} 6.0$ ) were added to the denatured sperm nuclei. After staining for $3 \mathrm{~min}$, samples were measured for green and red fluorescence using LSR II with a 488-nm excitation wavelength. For the SCSA assay, acid-treated sperm were stained with acridine orange, which emits red or green fluorescence when binding to single-stranded or double-stranded DNA, respectively. Sperm with impaired chromatin generate more single-stranded DNA after denaturation with acid treatment, and therefore emit more red fluorescence. DNA Fragmentation Index (DFI) is defined as the percentage of red/green + red fluorescence.

Transmission electron microscopy. Testes were fixed with $2 \%$ paraformaldehyde and $2 \%$ glutaraldehyde in $0.1 \mathrm{M}$ sodium phosphate buffer ( $\mathrm{pH} 7.4$ ), dehydrated and 
embedded in Epon. Sections were contrasted and imaged in a Hitachi H7000T transmission electron microscope.

Basic protein extraction and acid-urea gel electrophoresis. Testes were surgically decapsulated and homogenized in buffer (10 mM Tris- $\mathrm{HCl}(\mathrm{pH} 7.2), 0.32 \mathrm{M}$ sucrose, $5 \mathrm{mM} \mathrm{MgCl}_{2}, 0.1 \%$ Triton X-100 and $0.5 \mathrm{mM} \mathrm{PMSF}$ ). After centrifugation, cell pellets were resuspended in sonication buffer ( $10 \mathrm{mM}$ Tris- $\mathrm{HCl}$ ( $\mathrm{pH} 7.5)$, $25 \mathrm{mM}$ 2-mercaptoethanol) and sonicated using a Diagenode Bioruptor UCD-200 to obtain sonication-resistant spermatids, which represent step 12-16 spermatids. After centrifugation, sonication-resistant spermatid pellets were resuspended in $10 \mathrm{mM}$ Tris- $\mathrm{HCl}(\mathrm{pH}$ 7.5) by brief vortexing and $\mathrm{HCl}$ was added to a final concentration of $0.5 \mathrm{M}$. Samples were incubated on ice for $30 \mathrm{~min}$ to extract basic proteins. After centrifugation, the supernatant was transferred to a new tube and $20 \%$ trichloroacetic acid (final concentration) was added to precipitate basic proteins. Protein pellets were washed with acetone, dried and dissolved in buffer containing $5 \mathrm{M}$ urea, $0.5 \%$ acetic acid and $1 \% \beta$-2-mercaptoethanol. Proteins were separated by electrophoresis in acid-urea- $15 \%$ polyacrylamide gels and were subjected to either staining with Coomassie brilliant blue or western blot using the indicated antibodies.

TUNEL assay. TUNEL assays were performed with the In Situ Cell Death Detection Fluorescein Kit (Roche), following the manufacturer's instruction. Briefly, testes sections were deparaffinized, rehydrated and digested with $20 \mu \mathrm{g} \mathrm{ml}^{-1}$ Proteinase $\mathrm{K}$ in $10 \mathrm{mM}$ (Tris $\mathrm{pH} 7.5$ ) for $30 \mathrm{~min}$ at $37^{\circ} \mathrm{C}$. After washes, sections were incubated with TUNEL reaction mixture for $1 \mathrm{~h}$ at $37^{\circ} \mathrm{C}$, followed with lectin PNA $(1: 4,000,1 \mathrm{~h})$ and DAPI $(1: 5,000,5 \mathrm{~min})$ staining to visualize acrosomes and DNA, respectively.

ChIP. ChIP was performed using SimpleChIP Enzymatic Chromatin IP Kit (Cell Signaling) with the indicated antibodies. Primers for ChIP-qPCR are listed in Supplementary Table 5.

Centrifugal elutriation. Fractionation of spermatogenic cells through centrifugal elutriation was performed as previously described ${ }^{66}$ using a Beckman Coulter Avanti J-26XP centrifuge with JE-5.0 rotor.

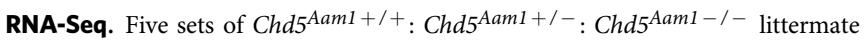
male mice with matched background and age ( $\sim 3$ months old) were used for elutriation. Round spermatids were purified through centrifugal elutriation of the testes pooled from five mice of the same genotype. Total RNA was prepared from the round spermatid samples using RNeasy kit (Qiagen) with DNase I treatment. RNA-Seq libraries were prepared from RNA samples using Illumina TruSeq protocol. RNA-Seq libraries were barcoded and sequenced on Illumina HiSeq 2000.

RNA-Seq data analysis. The quality of raw data was assessed and passed by FastQC. Reads were mapped to the $\mathrm{mm} 9$ reference genome with OLego (ref. 67). Cufflinks (v2.0.2) ${ }^{68}$ was used to estimate transcript expression levels represented by FPKM (fragments per kilo bases per million mapped reads) for all the samples. Ensembl transcripts annotation was provided $(-\mathrm{g})$ to guide transcriptome reconstruction. Cuffdiff (ref. 68) was run to detect differential expression between samples. Transcripts showing twofold or more expression changes in Chd5 $5^{\text {Aam } 1-1-}$ spermatids compared with Chd5 $5^{\text {Aam } 1+/+}$ spermatids were selected (false discovery rate $q=0.05$ ), and further filtered via hierarchical clustering using Genesis (v1.7.6) ${ }^{69}$. Clusters that either showed expression changes only in Chd5 $5^{\text {Aam } 1-/-}$ spermatids but not in Chd5 $5^{\text {Aam } 1+/-}$ spermatids, or those that showed gradual expression changes from Chd $5^{\text {Aam } 1+/+}$ spermatids to Chd5 $5^{\text {Aam 1 I / }}$ spermatids to Chd5 ${ }^{\text {Aam 1 - I- }}$ spermatids, were selected for GO analysis using DAVID $(\mathrm{v} 6.7)^{70}$.

qRT-PCR validation of RNA-Seq hits. qRT-PCR was first performed on the same RNA samples used for RNA-Seq, and then repeated using another set of RNA samples that were prepared from round spermatids that had been elutriationpurified from three different sets of Chd $5^{\text {Aam1+1+ }}$, Chd $5^{\text {Aam1+1- }}$ and Chd5 $5^{\text {Aam } 1-/-}$ littermate male mice with matched background and age. All qRT-PCR results were pooled and data are presented as mean \pm s.d. from four to five independent experiments. Primers for qRT-PCR and ChIP-qPCR are listed in Supplementary Table 5.

\section{References}

1. Hess, R. A. \& Renato de Franca, L. Spermatogenesis and cycle of the seminiferous epithelium. Adv. Exp. Med. Biol. 636, 1-15 (2008).

2. Tanaka, H. \& Baba, T. Gene expression in spermiogenesis. Cell. Mol. Life Sci. 62, 344-354 (2005).

3. Oakberg, E. F. Duration of spermatogenesis in the mouse and timing of stages of the cycle of the seminiferous epithelium. Am. J. Anat. 99, 507-516 (1956).
4. Oakberg, E. F. A description of spermiogenesis in the mouse and its use in analysis of the cycle of the seminiferous epithelium and germ cell renewal. Am. J. Anat. 99, 391-413 (1956).

5. Ahmed, E. A. \& de Rooij, D. G. Staging of mouse seminiferous tubule crosssections. Methods Mol. Biol. 558, 263-277 (2009).

6. Rousseaux, S. et al. Epigenetic reprogramming of the male genome during gametogenesis and in the zygote. Reprod. Biomed. Online 16, 492-503 (2008).

7. Hud, N. V., Allen, M. J., Downing, K. H., Lee, J. \& Balhorn, R. Identification of the elemental packing unit of DNA in mammalian sperm cells by atomic force microscopy. Biochem. Biophys. Res. Commun. 193, 1347-1354 (1993).

8. Ward, W. S. \& Coffey, D. S. DNA packaging and organization in mammalian spermatozoa: comparison with somatic cells. Biol. Reprod. 44, 569-574 (1991).

9. Bagchi, A. et al. CHD5 is a tumor suppressor at human 1p36. Cell 128, 459-475 (2007).

10. Paul, S. et al. Chd5 requires PHD-mediated histone 3 binding for tumor suppression. Cell Rep. 3, 92-102 (2013).

11. Oliver, S. S. et al. Multivalent recognition of histone tails by the PHD fingers of CHD5. Biochemistry 51, 6534-6544 (2012)

12. Vestin, A. \& Mills, A. A. The tumor suppressor Chd5 is induced during neuronal differentiation in the developing mouse brain. Gene Exp. Patterns 13, 482-489 (2013)

13. Berkovits, B. D. \& Wolgemuth, D. J. The role of the double bromodomaincontaining BET genes during mammalian spermatogenesis. Curr. Top. Dev. Biol. 102, 293-326 (2013).

14. Cocquet, J. et al. A genetic basis for a postmeiotic X versus $\mathrm{Y}$ chromosome intragenomic conflict in the mouse. PLoS Genet. 8, e1002900 (2012).

15. Skarnes, W. C. et al. A conditional knockout resource for the genome-wide study of mouse gene function. Nature 474, 337-342 (2011).

16. Zhuang, T. et al. CHD5 is required for spermiogenesis and chromatin condensation. Mech. Dev. 131, 35-46 (2014).

17. Bungum, M. Sperm DNA integrity assessment: a new tool in diagnosis and treatment of fertility. Obstet. Gynecol. Int. 2012, 531042 (2012).

18. Spiess, A. N. et al. Cross-platform gene expression signature of human spermatogenic failure reveals inflammatory-like response. Hum. Reprod. 22, 2936-2946 (2007).

19. Kimmins, S. \& Sassone-Corsi, P. Chromatin remodelling and epigenetic features of germ cells. Nature 434, 583-589 (2005).

20. Carrell, D. T. Epigenetics of the male gamete. Fertil. Steril. 97, 267-274 (2012)

21. Carrell, D. T., Emery, B. R. \& Hammoud, S. Altered protamine expression and diminished spermatogenesis: what is the link? Hum. Reprod. Update 13, 313-327 (2007).

22. Okada, Y., Scott, G., Ray, M. K., Mishina, Y. \& Zhang, Y. Histone demethylase JHDM2A is critical for Tnp1 and Prm1 transcription and spermatogenesis. Nature 450, 119-123 (2007).

23. Yu, Y. E. et al. Abnormal spermatogenesis and reduced fertility in transition nuclear protein 1-deficient mice. Proc. Natl Acad. Sci. USA 97, 4683-4688 (2000).

24. Zhao, M. et al. Targeted disruption of the transition protein 2 gene affects sperm chromatin structure and reduces fertility in mice. Mol. Cell Biol. 21, 7243-7255 (2001).

25. Awe, S. \& Renkawitz-Pohl, R. Histone H4 acetylation is essential to proceed from a histone- to a protamine-based chromatin structure in spermatid nuclei of Drosophila melanogaster. Syst. Biol. Reprod. Med. 56, 44-61 (2010).

26. Sonnack, V., Failing, K., Bergmann, M. \& Steger, K. Expression of hyperacetylated histone $\mathrm{H} 4$ during normal and impaired human spermatogenesis. Andrologia 34, 384-390 (2002).

27. Gaucher, J. et al. Bromodomain-dependent stage-specific male genome programming by Brdt. EMBO J. 31, 3809-3820 (2012).

28. Pivot-Pajot, C. et al. Acetylation-dependent chromatin reorganization by BRDT, a testis-specific bromodomain-containing protein. Mol. Cell. Biol. 23, 5354-5365 (2003).

29. Kramers, K. et al. Specificity of monoclonal anti-nucleosome auto-antibodies derived from lupus mice. J. Autoimmun. 9, 723-729 (1996).

30. Leduc, F., Maquennehan, V., Nkoma, G. B. \& Boissonneault, G. DNA damage response during chromatin remodeling in elongating spermatids of mice. Biol. Reprod. 78, 324-332 (2008).

31. Marcon, L. \& Boissonneault, G. Transient DNA strand breaks during mouse and human spermiogenesis new insights in stage specificity and link to chromatin remodeling. Biol. Reprod. 70, 910-918 (2004).

32. Sakkas, D. et al. Origin of DNA damage in ejaculated human spermatozoa. Rev Reprod. 4, 31-37 (1999).

33. Laberge, R. M. \& Boissonneault, G. On the nature and origin of DNA strand breaks in elongating spermatids. Biol. Reprod. 73, 289-296 (2005).

34. Steger, K. et al. Expression of mRNA and protein of nucleoproteins during human spermiogenesis. Mol. Hum. Reprod. 4, 939-945 (1998).

35. Morales, C. R., Kwon, Y. K. \& Hecht, N. B. Cytoplasmic localization during storage and translation of the mRNAs of transition protein 1 and protamine 1 , two translationally regulated transcripts of the mammalian testis. J. Cell Sci. 100(Pt 1): 119-131 (1991). 
36. Kleene, K. C. Patterns of translational regulation in the mammalian testis. Mol. Reprod. Dev. 43, 268-281 (1996).

37. Ling, T. et al. CHD4/NuRD maintains demethylation state of rDNA promoters through inhibiting the expression of the rDNA methyltransferase recruiter TIP5. Biochem. Biophys. Res. Commun. 437, 101-107 (2013).

38. Zentner, G. E. et al. CHD7 functions in the nucleolus as a positive regulator of ribosomal RNA biogenesis. Hum. Mol. Genet. 19, 3491-3501 (2010).

39. Bonisch, C. et al. H2A.Z.2.2 is an alternatively spliced histone H2A.Z variant that causes severe nucleosome destabilization. Nucleic Acids Res. 40, 5951-5964 (2012).

40. Tachiwana, H. et al. Structural basis of instability of the nucleosome containing a testis-specific histone variant, human H3T. Proc. Natl Acad. Sci. USA 107, 10454-10459 (2010).

41. Govin, J., Caron, C., Lestrat, C., Rousseaux, S. \& Khochbin, S. The role of histones in chromatin remodelling during mammalian spermiogenesis. Eur. J. Biochem. 271, 3459-3469 (2004).

42. Marfella, C. G. \& Imbalzano, A. N. The Chd family of chromatin remodelers. Mutat. Res. 618, 30-40 (2007).

43. Hall, J. A. \& Georgel, P. T. CHD proteins: a diverse family with strong ties. Biochem. Cell Biol. 85, 463-476 (2007).

44. Stanley, F. K., Moore, S. \& Goodarzi, A. A. CHD chromatin remodelling enzymes and the DNA damage response. Mutat. Res. 750, 31-44 (2013).

45. Egan, C. M. et al. CHD5 is required for neurogenesis and has a dual role in facilitating gene expression and polycomb gene repression. Dev. Cell 26, 223-236 (2013)

46. Boissonneault, G. Chromatin remodeling during spermiogenesis: a possible role for the transition proteins in DNA strand break repair. FEBS Lett. 514, 111-114 (2002).

47. Kierszenbaum, A. L. Transition nuclear proteins during spermiogenesis: unrepaired DNA breaks not allowed. Mol. Reprod. Dev. 58, 357-358 (2001).

48. Kim, Y., Fedoriw, A. M. \& Magnuson, T. An essential role for a mammalian SWI/SNF chromatin-remodeling complex during male meiosis. Development 139, 1133-1140 (2012).

49. Dowdle, J. A. et al. Mouse BAZ1A (ACF1) is dispensable for double-strand break repair but is essential for averting improper gene expression during spermatogenesis. PLoS Genet. 9, e1003945 (2013).

50. Lu, L. Y. et al. RNF8-dependent histone modifications regulate nucleosome removal during spermatogenesis. Dev. Cell 18, 371-384 (2010).

51. Sin, H. S. et al. RNF8 regulates active epigenetic modifications and escape gene activation from inactive sex chromosomes in post-meiotic spermatids. Genes Dev. 26, 2737-2748 (2012).

52. Dass, B. et al. Loss of polyadenylation protein tauCstF-64 causes spermatogenic defects and male infertility. Proc. Natl Acad. Sci. USA 104, 20374-20379 (2007).

53. Steger, K. Haploid spermatids exhibit translationally repressed mRNAs. Anat. Embryol. (Berl.). 203, 323-334 (2001).

54. Kleene, K. C. Poly(A) shortening accompanies the activation of translation of five mRNAs during spermiogenesis in the mouse. Development 106, 367-373 (1989).

55. Nomura, H. et al. WRNIP1 accumulates at laser light irradiated sites rapidly via its ubiquitin-binding zinc finger domain and independently from its ATPase domain. Biochem. Biophys. Res. Commun. 417, 1145-1150 (2012).

56. Hayashi, T. et al. Vertebrate WRNIP1 and BLM are required for efficient maintenance of genome stability. Genes Genet. Syst. 83, 95-100 (2008)

57. Tsurimoto, T., Shinozaki, A., Yano, M., Seki, M. \& Enomoto, T. Human Werner helicase interacting protein 1 (WRNIP1) functions as a novel modulator for DNA polymerase delta. Genes Cells 10, 13-22 (2005).

58. Morimoto, A. et al. A conserved KASH domain protein associates with telomeres, SUN1, and dynactin during mammalian meiosis. J. Cell Biol. 198, 165-172 (2012)

59. Gob, E., Schmitt, J., Benavente, R. \& Alsheimer, M. Mammalian sperm head formation involves different polarization of two novel LINC complexes. PLoS ONE 5, e12072 (2010).

60. Nery, F. C. et al. TorsinA binds the KASH domain of nesprins and participates in linkage between nuclear envelope and cytoskeleton. J. Cell Sci. 121, 3476-3486 (2008).
61. Ketema, M. et al. Requirements for the localization of nesprin-3 at the nuclear envelope and its interaction with plectin. J. Cell Sci. 120, 3384-3394 (2007).

62. Wilhelmsen, K. et al. Nesprin-3, a novel outer nuclear membrane protein, associates with the cytoskeletal linker protein plectin. J. Cell Biol. 171, 799-810 (2005).

63. Hammoud, S. S. et al. Distinctive chromatin in human sperm packages genes for embryo development. Nature 460, 473-478 (2009).

64. Adams, D. J. et al. Mutagenic insertion and chromosome engineering resource (MICER). Nat. Genet. 36, 867-871 (2004).

65. Pettitt, S. J. et al. Agouti C57BL/6N embryonic stem cells for mouse genetic resources. Nat. Methods 6, 493-495 (2009).

66. Zhao, M. et al. Utp14b: a unique retrogene within a gene that has acquired multiple promoters and a specific function in spermatogenesis. Dev. Biol. 304, 848-859 (2007).

67. Wu, J., Anczukow, O., Krainer, A. R., Zhang, M. Q. \& Zhang, C. OLego: fast and sensitive mapping of spliced mRNA-Seq reads using small seeds. Nucleic Acids Res. 41, 5149-5163 (2013).

68. Trapnell, C. et al. Transcript assembly and quantification by RNA-Seq reveals unannotated transcripts and isoform switching during cell differentiation. Nat. Biotechnol. 28, 511-515 (2010)

69. Sturn, A., Quackenbush, J. \& Trajanoski, Z. Genesis: cluster analysis of microarray data. Bioinformatics 18, 207-208 (2002).

70. Huang da, W., Sherman, B. T. \& Lempicki, R. A. Systematic and integrative analysis of large gene lists using DAVID bioinformatics resources. Nat. Protoc 4, 44-57 (2009).

71. Hess, R et al. in: Molecular Mechanisms in Spermatogenesis, Ch. 1 (Springer, 2008).

\section{Acknowledgements}

We thank W. Stephen Kistler for anti-Tnp antibodies, Jo H.M. Berden for antinucleosome antibodies, Emma Vernersson-Lindahl for critical reading of the manuscript and Lisa Bianco and Aigoul Nourjanova for technical assistance. This project was supported by the Office of the Director, National Institutes of Health under award numbers R01CA127383 (to A.A.M.) and R21OD018332 (to A.A.M.), NIH HG001696 (to M.Q.Z.) and NSFC 91019016 (to M.Q.Z.); the content is the sole responsibility of the authors. This work was performed with assistance from Cold Spring Harbour Laboratory Shared Resources, which are funded, in part, by the Cancer Center Support Grant 5P30CA045508.

\section{Author contributions}

W.L. and A.A.M. designed the experiments and prepared the manuscript with input from co-authors. W.L. and A.A.M. generated Chd5-deficient mouse models. W.L. performed experiments with assistance from S.-Y.K. for sperm analyses and IVF, with assistance from S.A.H. for electron microscopy, and with assistance from M.Z. and M.L.M. for centrifugal elutriation. W.L. and A.A.M. interpreted the data, with RNA-Seq data being analysed by J.W., W.L. and M.Q.Z

\section{Additional information}

Accession codes: RNA-seq data are deposited in the NCBI Short Read Archive under accession number SRPO40711.

Supplementary Information accompanies this paper at http://www.nature.com/ naturecommunications

Competing financial interests: The authors declare no competing financial interests.

Reprints and permission information is available online at http://npg.nature.com/ reprintsandpermissions/

How to cite this article: $\mathrm{Li}, \mathrm{W}$. et al. Chd5 orchestrates chromatin remodelling during sperm development. Nat. Commun. 5:3812 doi: 10.1038/ncomms4812 (2014). 